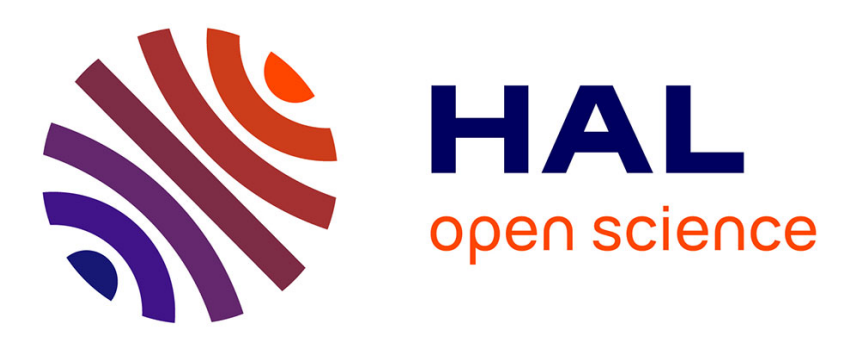

\title{
A Partitioned Solution Algorithm for Concurrent Computation of Stress-Strain and Fluid Flow in Continuous Casting Process
}

Shaojie Zhang, Gildas Guillemot, Charles-André Gandin, Michel Bellet

\section{- To cite this version:}

Shaojie Zhang, Gildas Guillemot, Charles-André Gandin, Michel Bellet. A Partitioned Solution Algorithm for Concurrent Computation of Stress-Strain and Fluid Flow in Continuous Casting Process. Metallurgical and Materials Transactions B, 2021, 52 (2), pp.978 - 995. 10.1007/s11663-021-02070-4 . hal-03193304

\section{HAL Id: hal-03193304 https://hal.science/hal-03193304}

Submitted on 9 Apr 2021

HAL is a multi-disciplinary open access archive for the deposit and dissemination of scientific research documents, whether they are published or not. The documents may come from teaching and research institutions in France or abroad, or from public or private research centers.
L'archive ouverte pluridisciplinaire HAL, est destinée au dépôt et à la diffusion de documents scientifiques de niveau recherche, publiés ou non, émanant des établissements d'enseignement et de recherche français ou étrangers, des laboratoires publics ou privés. 


\title{
A partitioned solution algorithm for concurrent computation of stress-strain and fluid flow in continuous casting process
}

\author{
Shaojie Zhang, Gildas Guillemot, Charles-André Gandin and Michel Bellet* \\ MINES ParisTech, PSL Research University, CEMEF - Centre de Mise en Forme des Matériaux, CNRS UMR \\ 7635, CS 10207, 1 rue Claude Daunesse, 06904 Sophia Antipolis cedex, France
}

Abstract: Control of macrosegregation phenomena and deformation related defects is a main issue in steel continuous casting. Numerical simulation could help industrial engineers to master these defects. However, as a first step, it is essential to achieve a concurrent computation of fluid flow in the bulk liquid and stress-strain evolution in the already solidified regions. With this aim in view, a new specific partitioned solver has been developed to model the liquid flow, essentially induced by the inlet jet distributed by the submerged nozzle, as well as the thermal deformation of the solid shell. The solver procedure allows simulating the transient regime, up to convergence to the steady-state regime. For this purpose, the computational finite element mesh moves and grows continuously. Within this evolving mesh, three different zones are defined: the solid shell as a pure Lagrangian zone, the liquid nozzle region as a pure Eulerian zone, and an intermediate Eulerian-Lagrangian zone. Conservation equations (energy, mass, and momentum) are solved in a general arbitrary Lagrangian-Eulerian framework, with a level-set formulation to track the free surface evolution at the meniscus. The article is composed of two parts. In the first part, the model is detailed with the resolution steps involved in the coupled resolution approach. In the second part, a simple verification test case is firstly proposed, followed by a more relevant and practical application to model an industrial pilot continuous casting process.

\section{*Corresponding author. Tel.: +33 4939574 61, michel.bellet@mines-paristech.fr.}
Email addresses: shaojie.zhang@mines-paristech.fr
(S. Zhang), gildas.guillemot@mines-paristech.fr (G. Guillemot), charles-andre.gandin@mines-paristech.fr
(C.-A. Gandin), michel.bellet@mines-paristech.fr (M. Bellet). 


\section{Introduction}

Continuous casting (CC) is likely the most important steel casting process due to its productivity and cost-efficiency. It is also a very sophisticated process as it involves numerous complex physical phenomena. Supported by the rapid development of computational capacities during the last decades, numerical modeling plays a more and more important role in the understanding and future developments of the CC process for steel companies. CC modeling consists principally in at least four physical phenomena: heat transfer, fluid flow, solid deformation, and solute transfer.

In the literature, most existing numerical models addressing $\mathrm{CC}$ can be classified into two categories. In the first category, the focus is set on thermo-fluid analyses, ignoring the other two aspects. The approach is essentially focused on what is named the primary cooling zone, that is the mold region where liquid steel is delivered at high speed by a submerged entry nozzle. With these models, industrial issues related to fluid flow can be investigated. Influence of process parameters such as the nozzle holes orientation, casting speed and mold width, on fluid flow patterns was successfully predicted ${ }^{[1,2]}$ Heat convection and other fluid flow related transport phenomena, for e.g. motion of argon gas bubbles and non-metallic inclusions, can be modeled. ${ }^{[3,4]}$ Complex phenomena, such as interactions between fluid flow, slag infiltration and mold oscillations are also considered. ${ }^{[5]}$ It is important to note that thermo-fluid simulation codes may give access to macrosegregation simulation by complementing the thermo-fluid solver with an additional solver for transport of chemical species. ${ }^{[6,7]}$ By contrast, in the second category, numerical models focus on solid deformation. The main investigated industrial issues are still in primary cooling: mold distortion and crack formation in solidified zones. But numerical codes for solid mechanics also permit studying the deformation of the solid shell during secondary cooling, with defects such as bulging between support rolls and associated crack formation. ${ }^{[8-10]}$

Following the presentation of these two categories - fluid flow and solid deformation - let us focus now on the interest in coupling the two approaches in the context of CC modeling:

- First, the deformation of the solid shell in primary cooling may modify significantly the thermal contact between the solid shell and the metallic mold, through the opening of a gas gap. This gap appearance decreases drastically the heat exchange coefficient characterizing the heat flow at product/mold interface. It is then easy to understand that an accurate simulation of the solidification process in the mold region must take into account such changes in thermal boundary conditions.

- Second, the prediction of the deformation and stress build-up in the solid shell in primary cooling is generally addressed by use of solid structural codes (such as Abaqus for instance). However, when proceeding this way, the influence of liquid convection loops in the mold region, with localized critical zones such as those directly impacted by the hot nozzle jet, cannot be taken into account. Hence, the prediction of cracking occurrence in the thin solid shell cannot be accurate and reliable. 
- Third, in secondary cooling, the interactions between the solid deformation and the subsequently induced fluid flow in the mushy zone have been proven to have a dominant effect on the formation of the central macrosegregation. ${ }^{[11]}$ Therefore, an algorithm allowing the concurrent computation of stress-strain and fluid-flow is essential for a successful modeling of such a phenomenon and furthermore to provide a more accurate model of solidification.

Regarding this coupling perspective, Thomas et al. have developed a strategy consisting in coupling several independent simulation codes together: one code for fluid flow, a second one for shell deformation. ${ }^{[12,13]}$ Zapulla et al. applied this approach to the simulation of the CC of stainless steel slabs, ${ }^{[12]}$ while Koric et al. applied it to beam blanks CC. ${ }^{[13]}$ It should be noted that such a coupling approach relies on a sophisticated interface engineering, in order to ensure the exchange of information between the different computational codes. This is all the more complex since this interface, namely the surface at solidus temperature, continuously evolve during the simulation. This might be also a possible source of robustness issues, or of efficiency loss in highly parallel computations. This is why the present approach is somewhat different, aiming at achieving such coupled solid/fluid resolutions in a unique computational code, and using a unique finite element mesh.

The coupled problem, as described above, can be seen as a general fluid-structure interaction (FSI) issue. However, contrary to most FSI problems, the interaction considered here is between a very stiff structure on one hand (the solid shell), and a low viscosity fluid on the other hand (the liquid metal). As pointed out by Heil et al. ${ }^{[14]}$ the coupling effect between solid and fluid mechanics in a FSI problem can be characterized by a FSI index, defined as the ratio of the flow stress in the fluid and solid regions. It was proven that for problems with a low FSI index, such as in the present problem for continuous casting, a partitioned approach is more efficient than a monolithic approach. By partitioned approach, one should understand a staggered resolution scheme in which separate fluid and solid problems are solved and coupled, while a monolithic approach is for a unique resolution. The reason for the best performance of the partitioned approach lies in the fact that the monolithic approach is affected by a loss of numerical conditioning. Indeed, the spectrum of eigenvalues of the set of equations to be solved is too wide and dramatically affects the convergence speed of iterative solvers. More detailed analysis can be found in references [14] and [15].

Another characteristic feature of solid/liquid coupling in the context of solidification is the coexistence of solid and liquid phases in mushy zones, where temperature is in between the liquidus and the solidus (or eutectic) temperatures corresponding to the solidification interval. At the process scale, the interface between the two phases cannot be explicitly modeled. Instead, the liquid and solid phases have to be considered in a homogenized way within a mushy zone. In the literature, there are only a few numerical models which are capable of coupling fluid flow and solid deformation. They were developed only in recent years. ${ }^{[16-18]}$ In those codes, fluid flow computation and stress-strain analysis are coupled and solved simultaneously within a single system of non-linear equations, expressing the momentum conservation equations relative to the solid and liquid phases, together with global mass conservation. 
They are not affected by numerical conditioning problems because they focus essentially on the mushy zone deformation, without involving very stiff constitutive equations for solid metal. Such constitutive equations derive from generalized non-Newtonian fluid behavior models. Some interesting results were obtained with this approach. Koshikawa et al. studied the fluid flow and associated macrosegregation induced by the lateral punching of an ingot deformed upon solidification, a specific solicitation aiming at mimicking soft reduction in CC. ${ }^{[1]}$ Fachinotti et al. ${ }^{[16]}$ and Rodrigues et al. ${ }^{[18]}$ directly addressed the simulation of soft reduction in CC. However, this approach is not retained in the present work for two reasons: i) using such a behavior model for the solid, it is impossible to address the prediction of residual stress in solid metal (as this requires elastic-(visco)plastic constitutive models such as the one described in the work of Bellet and Thomas ${ }^{[19]}$ ), and ii) the computational cost is prohibitive. Actually, solving for the liquid and solid velocity fields in a unique set requires that 7 unknown values should be determined at each node or cell of the computational grid: the 3 components of the solid velocity, the 3 components of the liquid velocity plus a pressure unknown in case of a mixed velocity-pressure formulation. Hence, 7 unknowns instead of 4 ( 3 velocity components plus a pressure) results in a dramatic increase of the computational time.

118 It is then clear that the partitioned approach is a good candidate to simulate FSI in CC. Similarly to the previous work aiming at simulating ingot casting, ${ }^{[15]}$ special care has to be put to consider the mushy zone in each of the two solvers. This is especially true in $\mathrm{CC}$, where the solid phase in the mushy zone is no more quasi-static, but moves at approximately the casting velocity. The global movement of the solid phase requires in turn an Arbitrary Lagrangian Eulerian (ALE) formulation. In the present work, a partitioned solution algorithm is then proposed for $\mathrm{CC}$ as an extension of the previous work done for ingot casting application. ${ }^{[15]}$ The numerical model principally consists of two resolution steps performed at each time increment on the same computational domain: the first one, labeled STEP I, is a solidoriented solution of the momentum and mass conservation equations, from which the stress-strain analysis is carried out in the regions partially or completely solidified. The second one, labeled STEP II, is a fluid-oriented solution of the momentum and mass conservation equations, giving access to the velocity and pressure fields in the fluid-containing zones, i.e. regions with liquid, mushy zone and gas. The volume averaging methodology and the Darcy's law are used to model the interactions between solid and liquid phases in the mushy zone. A characteristic feature of the present approach is that all conservation equations are formulated in the framework of a level set method in order to track the metal/gas interface. Another important point is that the partitioned algorithm is coupled with a nonlinear energy solver to calculate the temperature field. As this solver was initially developed by Saad et $a l .{ }^{[20]}$ under a fixed solid phase hypothesis, it is also extended to consider the movement of the solid phase.

137 The paper is organized as follows. In Section 2 the algorithm is described with a special focus on the 138 above-mentioned extensions. In Section 3, a verification of the extended approach is demonstrated 139 through a simple case test. It is followed by a more relevant and practical application to an industrial pilot continuous casting process. 


\section{$142 \quad 2$ Model description}

\section{$143 \quad 2.1 \quad$ Level set method: short reminder and notations}

144 A representative elementary volume (REV) of the simulation domain $\Omega$ is composed of two sub145 domains, as schematized in Fig. 1. A gas-subdomain $\Omega^{G}$ is defined above a metal sub-domain $\Omega^{M}$.

146 Three regions may be present in the metal sub-domain itself: a bulk liquid region, $l$, a fully solid region,

$147 s$, and a mushy zone made of a mixture of the two former regions, $l+s$. The level set method is used to 148 explicitly model the evolution of the metal/gas boundary during the continuous casting process, $\Gamma$. More 149 precisely, $\Gamma$ is represented by the zero-isovalue of the signed distance function $\varphi(\boldsymbol{x}, t)$, defined for any 150 point $\boldsymbol{x}$ and time $t$ in $\Omega$. The smoothed Heaviside function $\mathcal{H}^{M}$ is defined based on $\varphi(\boldsymbol{x}, t)$ as:

$$
\mathcal{H}^{M}(\varphi)=\left\{\begin{array}{cc}
0 & \text { if } \varphi<-\varepsilon \\
1 & \text { if } \varphi>\varepsilon \\
\frac{1}{2} & \left(1+\frac{\varphi}{\varepsilon}+\frac{1}{\pi} \sin \left(\frac{\pi \varphi}{\varepsilon}\right)\right) \text { if }-\varepsilon \leq \varphi \leq \varepsilon
\end{array}\right.
$$

151 where $\varepsilon$ is the half-thickness of the transition zone around the metal/gas boundary.

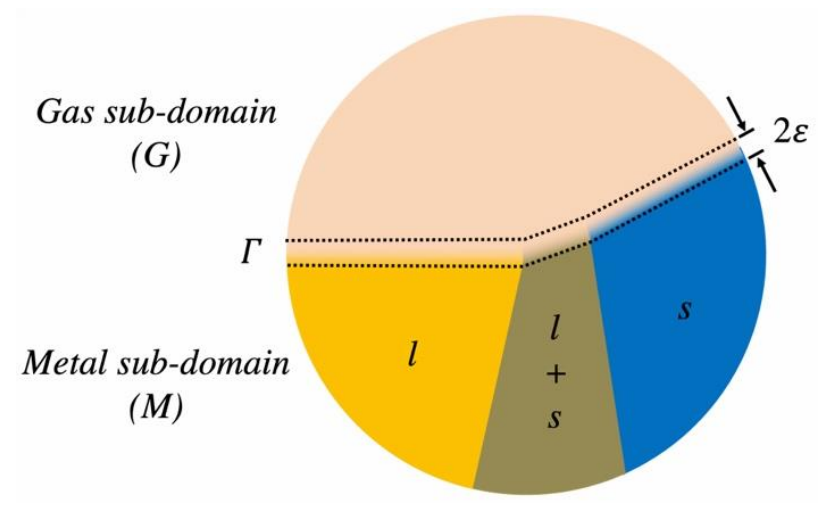

Fig.1. Schematic illustration of the simulation domain, including metal and gas sub-domains. The metal/gas boundary, $\Gamma$, is explicitly modeled through a diffusive level set transition zone, i.e. zone delimited by the two thick black dotted lines of thickness $2 \varepsilon$.

152 Respectively denoting $\psi^{M}$ and $\psi^{G}$ the physical property $\psi$ related to the metal and gas sub-domains,

153 the mixed property $\hat{\psi}$ is given by:

$$
\widehat{\psi}=\mathcal{H}^{M} \psi^{M}+\left(1-\mathcal{H}^{M}\right) \psi^{G}
$$

154 Thus, over the thickness $[-\varepsilon, \varepsilon]$ surrounding $\Gamma$, a smooth and continuous transition of properties is

155 defined. Note that the above-defined mixed property holds not only in the artificial transition zone 156 around the interface but also in the pure metal and gas sub-domains. Similarly, the mixed property $\hat{\psi}^{F}$ 157 associated with fluid, and being related to the liquid regions of the metal sub-domain and the gas sub158 domain, can be defined by the following expression:

$$
\widehat{\psi}^{F}=\mathcal{H}^{M} \psi^{l}+\left(1-\mathcal{H}^{M}\right) \psi^{G}
$$


where $\psi^{l}$ refers to the intrinsic property associated with the liquid phase in the metal sub-domain.

\subsection{Moving mesh, and ALE formulation}

162 The model of CC hereafter presented considers transient regimes. It aims at simulating non-steady states of the process thus requiring a continuously growing computational domain. The mold that characterizes the $\mathrm{CC}$ machine is of internal rectangular section. Its length defines the primary cooling region. The mold itself is not directly modeled. Instead, initial and boundary conditions of the simulation domain $\Omega$ are defined to account for its role in the solidification process. Initially, $\Omega$ encompasses a metal sub-domain $\Omega^{M}$ in the primary cooling region (i.e., in the mold), plus the above located gas sub-domain $\Omega^{G}$. During the simulation, $\Omega^{M}$ grows in the casting direction at the velocity defined by the withdrawal speed, $\boldsymbol{v}_{c c}$, progressively filling the whole primary cooling region, and then the secondary cooling zone underneath the mold.

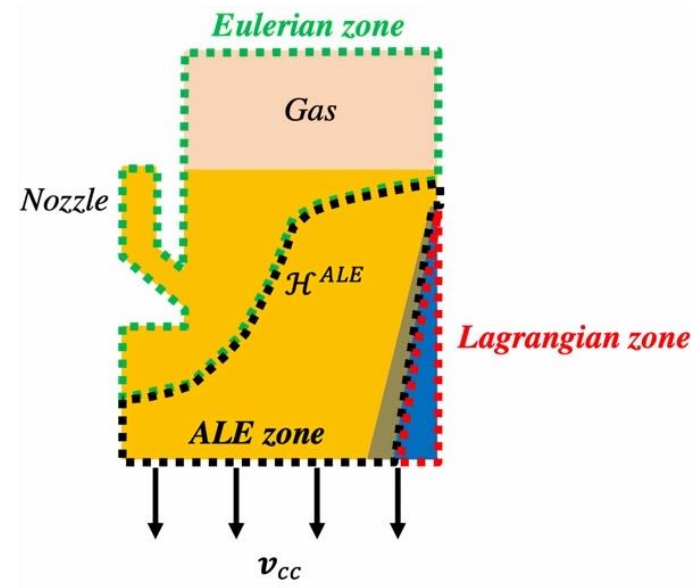

(a)

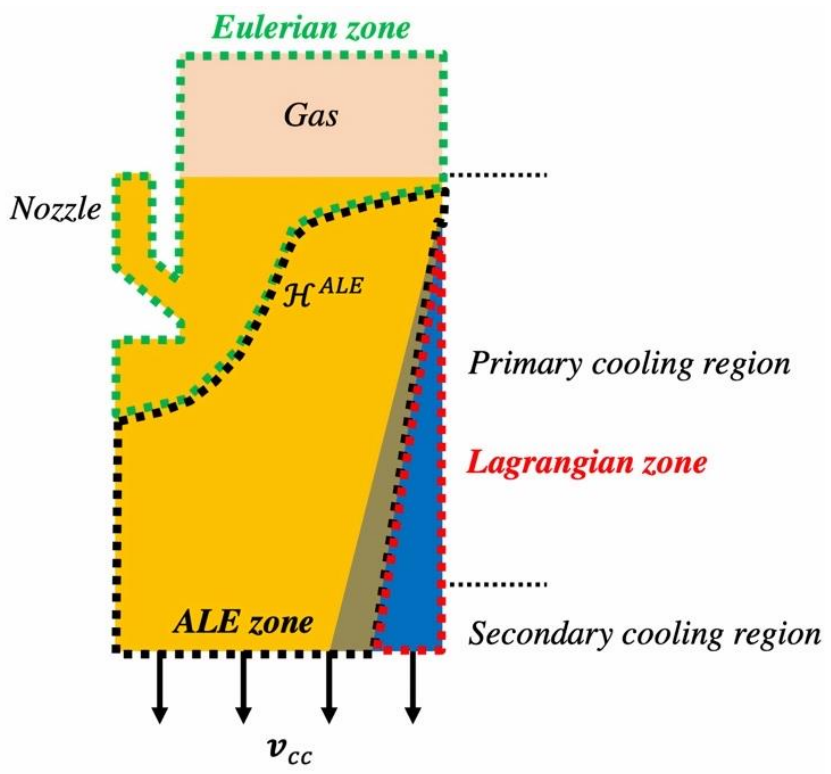

(b)

Fig.2. Schematics of the computational domain at (a) an early stage of the process when the metal is still fully located in the mold region and (b) at a later stage when the metal occupies the full mold region and has entered the secondary cooling region. Typical dimensions are: width 0.5 to $1 \mathrm{~m}$ (for half of the product, as represented here), thickness 150 to $250 \mathrm{~mm}$, height of the mold (primary cooling region) 600 to $800 \mathrm{~mm}$, thickness of solid shell at mold exit 10 to $15 \mathrm{~mm}$.

In this context, as explained hereafter, an evolving and constantly growing mesh is required. Fig. 2 illustrates the evolution of the computational domain $\Omega$ at two different stages of the simulation, respectively at an early stage (a) and at a later stage (b). At the top of the simulation domain, in $\Omega^{G}$ as well as in the neighborhood of the nozzle, a constant Eulerian zone is predefined where the mesh is fixed. The dimensions of this Eulerian zone are defined at the beginning of the simulation and kept unchanged all along the calculation. Its contour is drawn with thick green dotted lines in Fig. 2. In the solidified shell, a convenient and accurate approach consists in having a mesh following the displacement of the solid material. Indeed, the behavior of the solid material is elastic-viscoplastic, and 
thus of memory type. As it will be seen below, the solution of incremental elasto-viscoplasticity is based on total (or particle) time derivatives, which are extremely difficult to calculate accurately when using a non-Lagrangian mesh (i.e. a mesh that would not evolve at the same speed as the material). For this reason, a Lagrangian zone is defined, i.e. zone delimited by the contour using thick red dotted lines, as shown in Fig. 2, corresponding to the fully solidified regions in the metal sub-domain. Unlike the Eulerian zone, this Lagrangian zone keeps increasing during the simulation as it moves at material speed in the solid shell (Lagrangian approach). Note that the bottom boundary of the simulation domain grows at the casting speed with a vertical downward velocity, $\boldsymbol{v}_{c c}$. The rest of the computational domain is defined as an ALE zone, including the mushy zone and a part of the bulk liquid zone. The ALE zone is delimited by the thick black dotted contour in Fig. 2. Like the Lagrangian zone, this zone keeps increasing due to the imposed extraction speed of the machine which is prescribed along the bottom surface. The mesh movement in this zone is rather arbitrary.

In the context of this work, the transition between the ALE zone (mobile mesh) and the Eulerian zone (fixed mesh) is achieved through the use of a Heaviside function, $\mathcal{H}^{A L E}$. This Heaviside function is defined over the interface between the ALE and Eulerian zones, i.e. the coincident line of the thick dotted green and black lines shown in Fig. 2. This Heaviside function, varying between 0 in the Eulerian zone and 1 in the ALE zone, is smoothed over a certain thickness around the surface defined by $\mathcal{H}^{A L E}=$ 0.5 , that is the interface between the two zones. This allows a smooth and progressive evolution of the mesh dimensions that avoids excessive distortions. It should be mentioned that the location of this interface is rather arbitrary: it depends on the definition of the Eulerian zone. Nonetheless, once this interface is defined, it is kept unchanged over the whole simulation domain, and during the whole simulation, as well as the associated Heaviside function defined above. The strategy to force a smooth transition of the mesh evolution at the boundary between ALE and Lagrangian zones will be detailed in the next section. As a summary, the simulation considers an evolving computational domain, with a moving mesh composed of three regions:

- a fixed mesh in the gas and the nozzle neighborhood (Eulerian approach),

The mesh velocity will be denoted $\boldsymbol{v}_{m s h}$ in the sequel, the use of a moving mesh in turn inducing the use of an ALE formulation to take into account $\boldsymbol{v}_{m s h}$ in the discretization of conservation equations. In ALE, the relationship between the partial time derivative of any physical quantity $\psi$ with respect to the moving mesh, $\partial_{m s h} \psi / \partial t$ (often named grid derivative), and the total time derivative $d \psi / d t$, is resumed in the following equation:

$$
\frac{\partial_{m s h} \psi}{\partial t}=\frac{d \psi}{d t}-\left(\boldsymbol{v}-\boldsymbol{v}_{m s h}\right) \cdot \nabla \psi
$$

212 where $\boldsymbol{v}$ represents the material velocity field. Note that in the case of a pure Eulerian framework, $213 \boldsymbol{v}_{m s h} \equiv 0$ and $\partial_{m s h} \psi / \partial t=\partial \psi / \partial t$. The relationship between the partial and total derivatives of $\psi$ is 
215 simply expresses that $\partial_{m s h} \psi / \partial t=d \psi / d t$. Finally, substituting the classical relationship $d \psi / d t=$ $216 \partial \psi / \partial t+\boldsymbol{v} \cdot \nabla \psi$ into Eq. (4), an alternative relation is obtained:

$$
\frac{\partial_{m s h} \psi}{\partial t}=\frac{\partial \psi}{\partial t}+\boldsymbol{v}_{m s h} \cdot \nabla \psi
$$

Hence, this allows for an easy adaptation and discretization of conservation equations in ALE. First, in Lagrangian regions, it will be seen in Section 2.3 that only total derivatives are required. We have then $d \psi / d t \equiv \partial_{m s h} \psi / \partial t$, and the discretization is achieved by means of nodal finite differences: $\left(\psi_{n}^{t+\Delta t}-\psi_{n}^{t}\right) / \Delta t$, where $n$ denotes a node of the moving mesh. Second, in all other regions (metal in mushy or liquid state, gas) $\boldsymbol{v}_{m s h}$ is different from the material velocity, and possibly null. Besides, it will be seen in Sections 2.3 and 2.4 that the constitutive model is of no-memory type (viscoplastic or Newtonian behavior), and that conservation equations require only partial time derivatives $\partial \psi / \partial t$. They will be replaced by $\partial_{m s h} \psi / \partial t-\boldsymbol{v}_{m s h} \cdot \nabla \psi$, according to Eq. (5). The time grid derivatives will be discretized by means of nodal finite differences: $\left(\psi_{n}^{t+\Delta t}-\psi_{n}^{t}\right) / \Delta t$, where $n$ denotes a node of the moving mesh.

\subsection{STEP I: stress-strain analysis}

As already mentioned above, the two resolution steps are successively performed at each time increment.

230 An important point is that they are operated on the same computational mesh covering the entire domain

$231 \Omega$. Hence, STEP I is not operated on the "solid" only; STEP II is not operated on the "liquid" only.

232 Instead, both STEP I and STEP II are solved on $\Omega$.

233 The first resolution step is solid-oriented. This means that it essentially addresses the stress-strain 234 analysis in the solid regions and the mushy zone. The approach used was presented in detail in a previous 235 paper ${ }^{[15]}$ and is briefly reminded here. The following momentum and mass conservation equations are 236 solved for the velocity field $\boldsymbol{v}$ and the pressure field $p$, using a finite element method:

$$
\left\{\begin{array}{c}
\nabla \cdot \hat{\boldsymbol{s}}-\nabla p+\hat{\rho} \boldsymbol{g}=0 \\
\nabla \cdot \boldsymbol{v}=\mathcal{H}^{M}\left(H\left(T_{C}-T\right) \operatorname{tr}\left(\dot{\boldsymbol{\varepsilon}}_{e l}\right)+\operatorname{tr}\left(\dot{\boldsymbol{\varepsilon}}_{t h}\right)\right)
\end{array}\right.
$$

237 The tensor $\hat{\boldsymbol{s}}$ and the scalar $\hat{\rho}$ are respectively the mixed stress deviator and the mixed density in domain $238 \Omega$, as defined in Table 1. Vector $\boldsymbol{g}$ is the constant gravity vector.

\begin{tabular}{cc} 
Mixed property & Definition \\
\hline$\hat{\boldsymbol{s}}$ & $\mathcal{H}^{M} \boldsymbol{s}^{M}+\left(1-\mathcal{H}^{M}\right) \boldsymbol{s}^{G}$ \\
$\hat{\rho}$ & $\mathcal{H}^{M}\langle\rho\rangle^{M}+\left(1-\mathcal{H}^{M}\right) \rho^{G}$ \\
\hline
\end{tabular}

Table 1. Mixed properties between the metal and gas sub-domains in STEP I. 
$\boldsymbol{s}^{M}$ and $\langle\rho\rangle^{M}$ are respectively the stress tensor and average density in $\Omega^{M} . \boldsymbol{s}^{G}$ and $\rho^{G}$ are the stress tensor and density in $\Omega^{G}$. The average metal density $\langle\rho\rangle^{M}$ is defined as $g^{l}\langle\rho\rangle^{l}+g^{S}\langle\rho\rangle^{S}$ where $\langle\rho\rangle^{l}$ and $\langle\rho\rangle^{S}$

241 are respectively the intrinsic density of the liquid and solid phases with $g^{l}$ and $g^{s}$ corresponding

242 respectively to the local volume fractions of the liquid and solid phases.

$243 H\left(T_{C}-T\right)$ is the standard Heaviside function, taken for the temperature difference $\left(T_{C}-T\right)$. It is 244 introduced as an indicator relative to the use of a thermo-elastic-viscoplastic (TEVP) constitutive model 245 for elements in the metal sub-domain with an average temperature lower than a certain critical transition 246 temperature $T_{C}$, while a thermo-viscoplastic (TVP) model is used for elements in the metal sub-domain 247 with a temperature higher than $T_{C}$.

248 Constitutive equations of the TEVP model, below $T_{C}$, are described hereafter as given in reference [10]:

$$
\begin{gathered}
\dot{\boldsymbol{\varepsilon}}=\dot{\boldsymbol{\varepsilon}}_{e l}+\dot{\boldsymbol{\varepsilon}}_{v p}+\dot{\boldsymbol{\varepsilon}}_{t h} \\
\dot{\boldsymbol{\varepsilon}}_{e l}=\boldsymbol{E}^{-1} \dot{\boldsymbol{\sigma}}=\frac{1+v}{E} \dot{\boldsymbol{\sigma}}-\frac{v}{E} \operatorname{tr}(\dot{\boldsymbol{\sigma}}) \boldsymbol{I} \\
\dot{\boldsymbol{\varepsilon}}_{v p}=\frac{\sqrt{3}}{2 \bar{\sigma}}\left[\frac{\bar{\sigma}-\sigma_{Y}}{\sqrt{3} K \bar{\varepsilon}^{n}}\right]_{+}^{\frac{1}{m}} \boldsymbol{s} \\
\dot{\boldsymbol{\varepsilon}}_{t h}=-\frac{1}{3 \rho} \frac{d \rho}{d t} \mathbf{I}
\end{gathered}
$$

249 The strain rate tensor $\dot{\varepsilon}$ is split into an elastic part, $\dot{\boldsymbol{\varepsilon}}_{e l}$, a viscoplastic part, $\dot{\boldsymbol{\varepsilon}}_{v p}$, and a thermal part, $\dot{\boldsymbol{\varepsilon}}_{t h}$ 250 (Eq. (7)). The latter consists of the thermal expansion rate (Eq. (10)), with $\rho$ the density. Eq. (8) yields 251 the hypoelastic Hooke's law where $\boldsymbol{E}$ represents the elastic tensor depending on the Young's modulus $252 E$, and the Poisson's coefficient $v \cdot \boldsymbol{\sigma}$ denotes the total time derivative of the stress tensor. Eq. (9) gives 253 the relation between the viscoplastic strain rate tensor and the stress deviator $\boldsymbol{s}$. It is reminded here that 254 the stress deviator is defined as $\boldsymbol{s}=\boldsymbol{\sigma}-(1 / 3) \operatorname{tr}(\boldsymbol{\sigma}) \boldsymbol{I}=\boldsymbol{\sigma}+p \boldsymbol{I}$, where $\boldsymbol{\sigma}$ is the Cauchy stress tensor, $p$ 255 is the hydrostatic pressure, and $\boldsymbol{I}$ is the identity tensor. Coefficient $K$ is the viscoplastic consistency, $\sigma_{Y}$ 256 denotes the static yield stress below which no viscoplastic deformation occurs. The function $[x]_{+}$is 257 equal to 0 when $x$ is negative and to the value $x$ otherwise. Coefficients $m$ and $n$ denote the strain-rate 258 sensitivity coefficient, and the strain hardening coefficient, respectively. Finally, the corresponding 259 relationship between the von Mises stress, $\bar{\sigma}$, the generalized plastic strain, $\bar{\varepsilon}$,and the generalized strain 260 rate, $\dot{\bar{\varepsilon}}$, is given by:

$$
\bar{\sigma}=\sigma_{Y}+K(\sqrt{3})^{m+1} \dot{\bar{\varepsilon}}^{m} \bar{\varepsilon}^{n}
$$

261 Constitutive equations of the TVP model, over $T_{C}$, are written as follows:

$$
\begin{gathered}
\dot{\boldsymbol{\varepsilon}}=\dot{\boldsymbol{\varepsilon}}_{v p}+\dot{\boldsymbol{\varepsilon}}_{t h} \\
\dot{\boldsymbol{\varepsilon}}_{v p}=\frac{1}{2 K}(\sqrt{3} \dot{\bar{\varepsilon}})^{1-m} \boldsymbol{s}
\end{gathered}
$$




$$
\dot{\boldsymbol{\varepsilon}}_{t h}=-\frac{1}{3 \rho} \frac{d \rho}{d t} \boldsymbol{I}
$$

The strain rate tensor $\dot{\boldsymbol{\varepsilon}}$ is split into a viscoplastic part, $\dot{\boldsymbol{\varepsilon}}_{v p}$, and a thermal part, $\dot{\boldsymbol{\varepsilon}}_{t h}$ (Eq. (12)). Eq. (13) is the classical constitutive law for a generalized non-Newtonian fluid. It relates the viscoplastic strain rate $\dot{\boldsymbol{\varepsilon}}_{v p}$ to the stress deviator $\boldsymbol{s}$, in which the strain-rate sensitivity $m$ continuously increases with the liquid fraction in the mushy zone. The Newtonian behavior, which is assumed to be the behavior law for the liquid metal above its liquidus temperature, $T_{L}$, as well as for the gas, is obtained for $m=1$. In this case, the viscoplastic consistency $K$ is simply the dynamic viscosity of the fluid (liquid metal or gas). Finally, the corresponding relationship between the von Mises stress $\bar{\sigma}$ and the generalized strain rate $\dot{\bar{\varepsilon}}$ is the following one:

$$
\bar{\sigma}=K(\sqrt{3})^{m+1} \dot{\bar{\varepsilon}}^{m}
$$

In the present work, $T_{C}$ is taken exactly as the solidus temperature to model the TEVP behavior of steel in a fully solid state; the TVP model is used for metal either in the mushy state or liquid state. At this critical transition temperature, the continuity of the flow stress is obtained by taking $\sigma_{Y}\left(T_{C}\right)=0$ and $n\left(T_{C}\right)=0$.

\section{Specific points of STEP I resolution}

Two important characteristic features deserve attention:

- First, in order to prevent numerical instabilities due to the huge difference between solid consistency and liquid or gas viscosities, the values of these latter properties are artificially augmented. The liquid viscosity is typically set to $1 \mathrm{~Pa} \cdot \mathrm{s}$, which is about 200 times the nominal value for liquid steel for instance. The gas is considered as an incompressible Newtonian fluid in the gas sub-domain, also with a viscosity typically set to $1 \mathrm{~Pa} \cdot \mathrm{s}$. It is important to note that these simplifications have no significant impact on the main focus of STEP I, which is the calculation of stress and strain in already solidified regions during CC.

- A second important point in STEP I is the treatment of solidification shrinkage and liquid expansion. Usually, when performing the solid-oriented resolution step alone, without STEP II, like in reference [19], the thermal dilatation of both liquid and solid phases, are taken into consideration, together with the solidification shrinkage. However, when adding STEP II to STEP I, another strategy has been found more efficient. Keeping in mind that the focus of STEP I is the stress-strain analysis in already solidified regions, only the solid thermal expansion is taken into account below $T_{L}$ (considering $\langle\rho\rangle^{S}=f(T)$ as an input). Especially, in the mushy zone, we are essentially interested in the intrinsic velocity of the solid phase (i.e. the movement of the columnar dendritic structure). Such a numerical approximation appears to be a simple but efficient way to achieve the computation of a velocity field approaching the intrinsic velocity of the solid phase. In addition, over $T_{L}$, the material is considered incompressible: $\langle\rho\rangle^{M} \equiv\langle\rho\rangle^{S}\left(T_{L}\right)$. Hence, in Eq. (12), the thermal 
part of the strain rate tensor, $\dot{\varepsilon}_{t h}$, is constantly null. The treatment of solidification shrinkage and liquid expansion is done in STEP II and explained in the next Section.

296 Finally, a velocity-pressure resolution of the weak form of Eq. (6) is performed on $\Omega$. The velocity and 297 pressure fields resulting from this first step are denoted $\left(\boldsymbol{v}_{I}, p_{I}\right)$.

\subsection{STEP II: fluid flow computation}

300 In this second fluid-oriented resolution step, the solution from the previous STEP I is taken as an entry. 301 The objective of this second step resolution is to calculate the fluid flow in liquid and mushy regions, 302 taking into account the motion of the solid phase, as deduced from STEP I. In order to consider liquid 303 flow through the permeable solid phase in the mushy zone, an effective two-phase approach is used with 304 a volume-averaged method. ${ }^{[21]}$ Solidification is assumed to take place with a purely columnar structure. 305 Interactions between solid and liquid phases in the mushy zone are modeled by the Darcy's law with a 306 permeability coefficient $\kappa$ approximated by the Carman-Kozeny relationship. ${ }^{[22]}$ Although the liquid is 307 considered as incompressible with a Newtonian behavior, a compressible formulation is used to deal 308 with solidification shrinkage in the mushy zone and thermal dilatation of the liquid phase. It is also 309 important to mention that in STEP II, contrary to STEP I, the nominal liquid viscosity is now used.

310 In a previous paper, ${ }^{[15]}$ the conservation equations governing the fluid-oriented problem have been 311 established in the context of a level set formulation applied to a static mesh. As mentioned in Section 3122.2 , its extension to the context of a mobile mesh is achieved by replacing the partial time derivatives 313 by the grid derivatives according to Eq. (5). The conservation equations for STEP II are now expressed 314 with the ALE formulation:

$$
\left\{\begin{array}{c}
\hat{\rho}_{0}^{F}\left(\frac{\partial_{m s h} \boldsymbol{v}}{\partial t}+\frac{1}{\hat{g}^{F}}(\nabla \boldsymbol{v})\left(\boldsymbol{v}-\hat{g}^{F} \boldsymbol{v}_{m s h}\right)\right)-\nabla \cdot \widehat{g \boldsymbol{s}}^{F}+\hat{g}^{F} \nabla p-\widehat{\boldsymbol{g \rho}}^{F} \boldsymbol{g} \\
+\hat{g}^{F} \hat{\mu}^{F}\left(\hat{\kappa}^{F}\right)^{-1}\left(\boldsymbol{v}-\boldsymbol{v}_{I}\right)=0 \\
\nabla \cdot \boldsymbol{v}=-\frac{\mathcal{H}^{M}}{\langle\rho\rangle^{l}}\left(\frac{\partial_{m s h}\langle\rho\rangle^{M}}{\partial t}+\boldsymbol{v} \cdot \nabla\langle\rho\rangle^{l}-\boldsymbol{v}_{m s h} \cdot \nabla\langle\rho\rangle^{M}+\nabla \cdot\left(g^{S}\langle\rho\rangle^{S} \boldsymbol{v}_{I}\right)\right)
\end{array}\right.
$$

315 where the unknowns are the average fluid velocity, $\boldsymbol{v}=\mathcal{H}^{M} g^{l}\langle\boldsymbol{v}\rangle^{l}+\left(1-\mathcal{H}^{M}\right)\langle\boldsymbol{v}\rangle^{G}$, and the pressure, $316 p$. Notations $\hat{\rho}_{0}^{F}, \widehat{g}^{F}, \widehat{g s}^{F}, \widehat{g \rho}^{F}, \hat{\mu}^{F}, \hat{\kappa}^{F}$ correspond respectively to the mixing properties of the fluid 317 density, the fluid fraction, the averaged fluid stress deviator, the averaged fluid density with temperature 318 dependence, the fluid viscosity and the fluid permeability. These variables are defined in Table 2. 


\begin{tabular}{cc} 
Mixed property & Definition \\
\hline$\hat{g}^{F}$ & $\mathcal{H}^{M} g^{l}+\left(1-\mathcal{H}^{M}\right)$ \\
$\hat{\rho}_{0}^{F}$ & $\mathcal{H}^{M}\langle\rho\rangle_{0}^{l}+\left(1-\mathcal{H}^{M}\right) \rho^{G}$ \\
$\widehat{g \rho}^{F}$ & $\mathcal{H}^{M} g^{l}\langle\rho\rangle^{l}+\left(1-\mathcal{H}^{M}\right) \rho^{G}$ \\
& with $\langle\rho\rangle^{l}=\langle\rho\rangle_{0}^{l}\left(1-\beta_{T}^{l}\left(T-T_{L}\right)\right)$ \\
$\widehat{g s}^{F}$ & $\mathcal{H}^{M} g^{l}\langle s\rangle^{l}+\left(1-\mathcal{H}^{M}\right) \boldsymbol{s}^{G}$ \\
$\hat{\mu}^{F}$ & $\mathcal{H}^{M} \mu^{l}+\left(1-\mathcal{H}^{M}\right) \mu^{G}$ \\
$\widehat{\kappa}^{F}$ & $\lambda_{2}{ }^{2} \hat{g}^{F^{3}} /\left(180\left(1-\hat{g}^{F}\right)^{2}\right)$ \\
\hline
\end{tabular}

Table 2. Mixed properties between the liquid regions of the metal sub-domain and the gas sub-domain in STEP II.

$\langle\rho\rangle_{0}^{l},\langle\rho\rangle^{l}, \beta_{T}^{l},\langle\boldsymbol{s}\rangle^{l}, \mu^{l}, \lambda_{2}$ are respectively the intrinsic reference liquid density at $T_{L}$, the intrinsic liquid density depending linearly from the temperature, the dilatation coefficient of the liquid, the intrinsic liquid stress tensor, the liquid viscosity, and the secondary dendrite arm spacing. $\mu^{G}$ is the viscosity of the gas, assumed to be Newtonian and incompressible.

323 In the fully solid regions, there is actually no need to operate a STEP II resolution: all relevant information (velocity field, updated values of the generalized viscoplastic deformation, deviatoric stress tensor, and pressure) has been already calculated in the first solid-oriented resolution step. However, in the present approach, STEP II is operated on the whole domain $\Omega$, but with a Dirichlet condition applied to the fully solid regions: for nodes with their nodal liquid fraction $g^{l}$ equal to zero, $\boldsymbol{v}_{I I}$ is imposed equal to $\boldsymbol{v}_{I}$. Finally, Eq. (16) is solved with a stabilized SUPG-PSPG finite element method. ${ }^{[23]}$ The velocity and pressure fields resulting from this second step are denoted $\left(\boldsymbol{v}_{I I}, p_{I I}\right)$.

\subsection{Coupled thermal resolution}

332 A non-linear energy solver is coupled with the above STEP I and STEP II to provide the temperature 333 field during the continuous casting process. The conventional energy conservation equation for 334 solidification problems is given in Eq. (17), averaged over the REV in the metal sub-domain:[21]

$$
\frac{\partial\langle\rho h\rangle^{M}}{\partial t}+\nabla \cdot\langle\rho h \boldsymbol{v}\rangle^{M}-\nabla \cdot\left(\langle k\rangle^{M} \nabla T\right)=0
$$

where $\langle\rho h\rangle^{M}=g^{l}\langle\rho\rangle^{l}\langle h\rangle^{l}+g^{S}\langle\rho\rangle^{S}\langle h\rangle^{s},\langle\rho h \boldsymbol{v}\rangle^{M}=g^{l}\langle\rho\rangle^{l}\langle h\rangle^{l}\langle\boldsymbol{v}\rangle^{l}+g^{S}\langle\rho\rangle^{S}\langle h\rangle^{S}\langle\boldsymbol{v}\rangle^{S}$ and $\langle k\rangle^{M}=$ $g^{s}\langle k\rangle^{s}+g^{l}\langle k\rangle^{l}$ with $\langle h\rangle^{l},\langle h\rangle^{s},\langle k\rangle^{l},\langle k\rangle^{s},\langle\boldsymbol{v}\rangle^{l}$ and $\langle\boldsymbol{v}\rangle^{s}$ corresponding respectively to the intrinsic specific enthalpy, the intrinsic heat conductivity and the intrinsic velocity relative to the liquid and solid phases, respectively. Based on the relationship given in Eq. (5), the ALE formulation of Eq. (17) is given by: 


$$
\frac{\partial_{m s h}\langle\rho h\rangle^{M}}{\partial t}+\nabla \cdot\langle\rho h \boldsymbol{v}\rangle^{M}-\boldsymbol{v}_{m s h} \cdot \nabla\langle\rho h\rangle^{M}-\nabla \cdot\left(\langle k\rangle^{M} \nabla T\right)=0
$$

Developing the expressions of $\langle\rho h v\rangle^{M}$ and $\langle\rho h\rangle^{M}$, we obtain:

$$
\begin{gathered}
\frac{\partial_{m s h}\langle\rho h\rangle^{M}}{\partial t}+\nabla \cdot\left(g^{l}\langle\rho\rangle^{l}\langle h\rangle^{l}\langle\boldsymbol{v}\rangle^{l}\right)-\boldsymbol{v}_{m s h} \cdot \nabla\left(g^{l}\langle\rho\rangle^{l}\langle h\rangle^{l}\right) \\
+\nabla \cdot\left(g^{s}\langle\rho\rangle^{s}\langle h\rangle^{s}\langle\boldsymbol{v}\rangle^{s}\right)-\boldsymbol{v}_{m s h} \cdot \nabla\left(g^{s}\langle\rho\rangle^{s}\langle h\rangle^{s}\right)-\nabla \cdot\left(\langle k\rangle^{M} \nabla T\right)=0
\end{gathered}
$$

341 Let's first consider the following term, $\nabla \cdot\left(g^{s}\langle\rho\rangle^{s}\langle h\rangle^{s}\langle\boldsymbol{v}\rangle^{s}\right)-\boldsymbol{v}_{m s h} \cdot \nabla\left(g^{s}\langle\rho\rangle^{s}\langle h\rangle^{s}\right)$ in Eq. (19). This term represents the energy transport related to the solid phase. Assuming that the volume change of the solid, due to elasticity and thermal dilatation, has a negligible impact on heat transfer, the solid phase is thus assumed here to be intrinsically incompressible, $\nabla \cdot\langle\boldsymbol{v}\rangle^{S}=0$. Therefore, the above term is simplified into the following form, $\left(\langle\boldsymbol{v}\rangle^{s}-\boldsymbol{v}_{m s h}\right) \cdot \nabla\left(g^{S}\langle\rho\rangle^{S}\langle h\rangle^{S}\right)$. Note that in the bulk liquid this term is zero. Besides, the fully solidified regions are considered as Lagrangian in the present ALE framework. Therefore, in fully solidified regions, $\boldsymbol{v}_{m s h}=\langle\boldsymbol{v}\rangle^{S}$ and this term also reduces to zero.

Finally, in the specific context of the present work, the mesh velocity $\boldsymbol{v}_{m s}$ in the mushy zone is proposed with the following explicit form:

$$
\boldsymbol{v}_{m s h}=g^{l} \boldsymbol{v}_{c c}+g^{s} \boldsymbol{v}_{I}
$$

where $\boldsymbol{v}_{I}$ is the solution field from the previous solid-oriented resolution step and $\boldsymbol{v}_{c c}$ the constant casting velocity of the CC machine. We remind that $\boldsymbol{v}_{I}$ is designed to approach the intrinsic solid velocity $\langle\boldsymbol{v}\rangle^{S}$ in the previous solid-oriented resolution step. Besides, in continuous casting $\langle\boldsymbol{v}\rangle^{S}$ in the mushy zone is nearly $\boldsymbol{v}_{c c}$ due to the continuity of the solid phase between the mushy zone and fully solidified shell in contact with the molds. By consequence, the above-defined $\boldsymbol{v}_{m s h}$ keeps being a good approximation of the intrinsic solid velocity $\langle\boldsymbol{v}\rangle^{s}$ in the mushy zone. Therefore, this first energy transport term related to the solid phase, $\left(\langle\boldsymbol{v}\rangle^{s}-\boldsymbol{v}_{m s h}\right) \cdot \nabla\left(g^{s}\langle\rho\rangle^{s}\langle h\rangle^{s}\right)$, finally reduces to zero in the whole metal sub-domain, including the bulk liquid, the mushy zone and the fully solidified regions, and this term can be fully neglected from Eq. (19). In other words, the energy transport due to the motion of the solid phase in the present model will be achieved through the mesh updating process within the ALE framework. Finally, it is worth noting that the above-defined mesh velocity also holds in the fully solidified regions, i.e. $g^{s}=1$ and $\boldsymbol{v}_{m s}$ recovers the material speed in the solid shell, $\boldsymbol{v}_{I}$. A smooth transition of mesh is thus ensured at the boundary of ALE and Lagrangian zones.

As stated above, the definition of the mesh velocity in the fully liquid zone is rather arbitrary and may be different according to the specific configuration of each simulation. As explained in Section 2.2, a pure Eulerian zone is usually defined with $\boldsymbol{v}_{m s h}=0$ in regions where metal remains in a liquid state, for e.g. regions near the nozzle entry. More intuitive details of such a definition will be given later in application to the $\mathrm{CC}$ process. Indeed, an optimized mesh velocity would be better to prevent mesh distortions, such as the algorithm described in the work of Bellet and Fachinotti, ${ }^{[10]}$ where the mesh 
velocity of a node in the liquid zone was defined roughly as the average velocity of its neighboring nodes.

371 Considering now the second term $\nabla \cdot\left(g^{l}\langle\rho\rangle^{l}\langle h\rangle^{l}\langle\boldsymbol{v}\rangle^{l}\right)-\boldsymbol{v}_{m s h} \cdot \nabla\left(g^{l}\langle\rho\rangle^{l}\langle h\rangle^{l}\right)$ in Eq. (19), it represents 372 the energy transport related to the liquid phase. Assuming that the energy transfer due to the solidification shrinkage and the thermal dilatation of the liquid phase is negligible, this term can be approximated by $g^{l}\langle\boldsymbol{v}\rangle^{l} \nabla\left(\langle\rho\rangle^{l}\langle h\rangle^{l}\right)-\boldsymbol{v}_{m s h} \cdot \nabla\left(g^{l}\langle\rho\rangle^{l}\langle h\rangle^{l}\right)$. We remind that $\boldsymbol{v}_{I I}$, the solution from the previous fluid-oriented resolution step, represents the averaged liquid velocity, $g^{l}\langle\boldsymbol{v}\rangle^{l}$, in the liquid and mushy zones. In the meantime, it also tends to the solid velocity $\boldsymbol{v}_{I}$ when approaching the end of the mushy zone due to the Dirichlet condition applied to the fully solidified regions. Therefore, the following approximation is proposed for the above energy transport term related to the liquid phase:

$$
g^{l}\langle\boldsymbol{v}\rangle^{l} \cdot \nabla\left(\langle\rho\rangle^{l}\langle h\rangle^{l}\right)-\boldsymbol{v}_{m s h} \cdot \nabla\left(g^{l}\langle\rho\rangle^{l}\langle h\rangle^{l}\right)=\left(\boldsymbol{v}_{I I}-\boldsymbol{v}_{m s h}\right) \cdot \nabla\left(\langle\rho\rangle^{l}\langle h\rangle^{l}\right)
$$

Note that in the bulk liquid, $\boldsymbol{v}_{I I}=g^{l}\langle\boldsymbol{v}\rangle^{l}$, the right-hand side (RHS) term of Eq. (21) recovers exactly the left-hand-side (LHS) term of Eq. (21). In the fully solidified regions, the LHS term is zero as $g^{l}=0$ and RHS term is also zero as both $\boldsymbol{v}_{I I}$ and $\boldsymbol{v}_{m s h}$ equal to $\boldsymbol{v}_{I}$. Besides, in the mushy zone, Eq. (21) remains a quite reasonable approximation, especially in regions with high liquid fraction where the numerical solution $\boldsymbol{v}_{I I}$ corresponds nearly to $g^{l}\langle\boldsymbol{v}\rangle^{l}$. Finally, assuming that contribution of the gradient of the liquid density is negligible in Eq. (21) and taking into consideration the enthalpy relationship relative to the liquid phase, the above equation becomes:

$$
\left(\boldsymbol{v}_{I I}-\boldsymbol{v}_{m s h}\right) \cdot \nabla\left(\langle\rho\rangle^{l}\langle h\rangle^{l}\right)=\left(\boldsymbol{v}_{I I}-\boldsymbol{v}_{m s h}\right) \cdot\langle\rho\rangle^{l}\left\langle C_{p}\right\rangle^{l} \nabla T
$$

where $\left\langle C_{p}\right\rangle^{l}$ is the intrinsic specific heat of the liquid phase defined by the liquid enthalpy $\langle h\rangle^{l}=$ $\left\langle C_{p}\right\rangle^{l} T+L_{f}$ with constant latent heat of fusion $L_{f}$. Considering the above approximations made over the energy transport term relative to both solid and liquid phases, the energy conservation equation in the metal sub-domain for the CC process under the ALE framework is given by:

$$
\frac{\partial_{m s h}\langle\rho h\rangle^{M}}{\partial t}+\left(\boldsymbol{v}_{I I}-\boldsymbol{v}_{m s h}\right) \cdot\langle\rho\rangle^{l}\left\langle C_{p}\right\rangle^{l} \nabla T-\nabla \cdot\left(\langle k\rangle^{M} \nabla T\right)=0
$$

Finally, the energy conservation equation is developed under a level set formulation and is given by:

$$
\frac{\partial_{m s h} \widehat{\rho h}}{\partial t}+\left(\boldsymbol{v}_{I I}-\boldsymbol{v}_{m s h}\right) \cdot \widehat{\rho C}_{p}^{F} \nabla T-\nabla \cdot(\hat{k} \nabla T)=0
$$

with $\widehat{\rho h}, \widehat{\rho C_{p}}, \hat{k}$ corresponding respectively to the mixing properties of the volumetric enthalpy, the volumetric heat capacity and the heat conductivity between the metal and gas sub-domains, defined in Table 3. 


\begin{tabular}{cc}
\hline Mixed property & Definition \\
\hline$\widehat{\rho h}$ & $\mathcal{H}^{M}\langle\rho h\rangle^{M}+\left(1-\mathcal{H}^{M}\right) \rho^{G} h^{G}$ \\
$\widehat{\rho C}_{p}{ }^{F}$ & $\mathcal{H}^{M}\langle\rho\rangle^{l}\left\langle C_{p}\right\rangle^{l}+\left(1-\mathcal{H}^{M}\right) \rho^{G} C_{p}^{G}$ \\
$\hat{k}$ & $\mathcal{H}^{M}\langle k\rangle^{M}+\left(1-\mathcal{H}^{M}\right) k^{G}$ \\
\hline
\end{tabular}

Table 3. Mixed properties between the metal and gas sub-domains in the coupled heat transfer step.

394 where $h^{G}, C_{p}^{G}$ and $k^{G}$ are respectively the specific enthalpy, the specific heat and the heat conductivity 395 of the gas. It is worth noting that Eq. (24) holds for both continuous and ingot casting processes. 396 Especially, in a fully Eulerian framework where $\boldsymbol{v}_{m s h}=0$, Eq. (24) recovers the previous energy 397 conservation equation developed for ingot casting processes under a fixed-solid hypothesis. ${ }^{[15]}$

\subsection{Algorithm scheme}

400 The algorithm scheme of the present partitioned solution algorithm for continuous casting is presented,

401 considering that the two resolutions, STEP I and STEP II, and the coupled thermal resolution are 402 performed once at each time increment $\Delta t$. The incremental resolution scheme is divided into 7 modules, 403 as detailed hereunder in Fig.3:

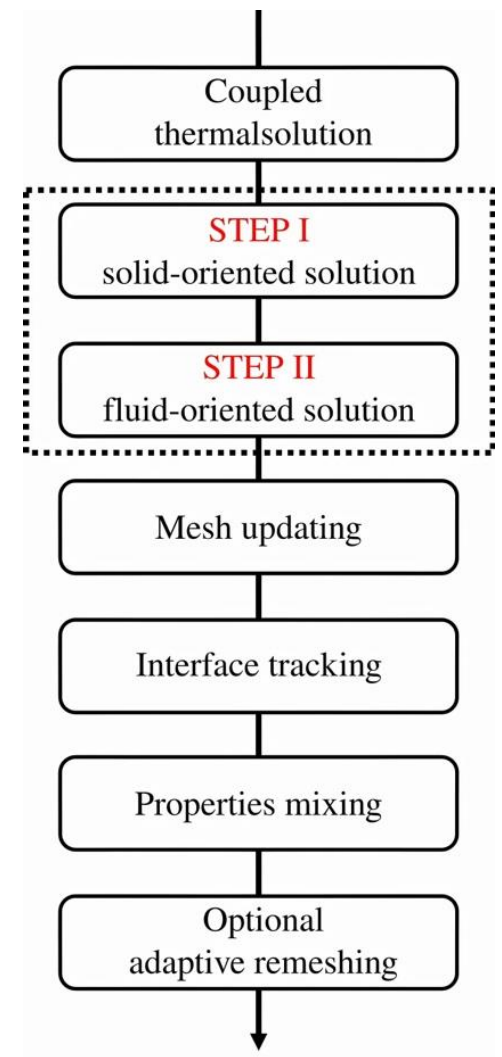

Fig.3. Resolution scheme in a time increment 
- Coupled thermal solution. The energy conservation equation is solved, giving access to the temperature distribution in the metal and gas sub-domains, and to the liquid metal fraction.

- STEP I, solid-oriented solution. The first folder of the mass and momentum conservation equations focuses on the stress-strain analysis in the already solidified regions, with an augmented liquid viscosity and a continuity of solid density in the mushy and liquid regions. It provides velocity and pressure fields on the whole domain: $\left(\boldsymbol{v}_{I}, p_{I}\right)$. However, only $\boldsymbol{v}_{I}$ at nodes belonging to fully solid elements and in the mushy zone will be used in the follow-up of the resolution scheme. The stress tensor $\sigma$, and the associated generalized plastic strain $\bar{\varepsilon}$ and strain rate $\dot{\bar{\varepsilon}}$, are also deduced from this step.

- STEP II fluid-oriented solution. The second folder of the mass and momentum conservation equations consists of the fluid flow computation in the liquid and mushy regions, with a real liquid viscosity and including solidification shrinkage. It provides velocity and pressure fields on the whole domain: $\left(\boldsymbol{v}_{I I}, p_{I I}\right)$. Note that at nodes belonging to fully solid elements, $\boldsymbol{v}_{I I}$ is imposed equal to $\boldsymbol{v}_{I}$.

- Mesh updating. The position of each mesh node is updated following an explicit scheme with the mesh velocity, $\boldsymbol{v}_{m s h}$ :

$$
x^{\text {new }}=x^{\text {old }}+\Delta t v_{m s h}
$$

It should be reminded that the energy transportation relative to the solid phase in the solid and mushy zones, is achieved through this mesh updating process under the present ALE framework.

- Metal/gas interface tracking. The updating of the level set function permits interface tracking. It is achieved by the convection-reinitialization scheme. ${ }^{[24]}$ The advection velocity field $\boldsymbol{v}$ is equal to the fluid velocity $\boldsymbol{v}_{I I}$ deduced from STEP II.

- Mixing of material properties according to the value of the updated level set function.

- Possible adaptive remeshing guided either by error estimation for different solution fields, as proposed by Coupez, ${ }^{[25]}$ or more simply formulated based on signed level set distance function.

\section{Simulation results}

\section{$430 \quad 3.1 \quad$ Verification test case}

431 The objective of the test case detailed hereafter is to check the correct implementation and the 432 performance of the above-proposed partitioned solution algorithm, including the new features 433 introduced in the thermal solver, i.e. introduction of the solid movement, the energy transport relative 434 to the solid phase and the mesh velocity. For this specific purpose, a simple solidification process configuration is studied, in which the gas sub-domain is neglected, as the new developments mainly 
436 affect the metal sub-domain. Besides, the solid-oriented step is oversimplified: no effective stress-strain 437 analysis is performed. Only the velocity of the solid phase is imposed, its value being equal to the 438 constant casting speed of the machine.

\section{$440 \quad$ 3.1.1 Model description}

441 The test case consists of the solidification of two identical 3D parallelepipedic ingots, under the same 442 heat boundary condition. The first ingot is fixed and serves as the reference simulation. Its solidification 443 is simulated using the previous algorithm developed for casting process. ${ }^{[15]}$ The second ingot is entirely 444 submitted to a constant downward movement and is simulated with the algorithm presented above. For 445 simplicity, only the configuration of the moving ingot simulation is presented here as shown in Fig.4. $446 v_{c s t}$ denotes the value of the downward moving velocity. The initial mesh is isotropic, defined with a 447 uniform mesh size of $1 \mathrm{~mm}$. A constant mesh velocity $\boldsymbol{v}_{m s h}$ is defined over the whole ingot, oriented 448 downward and aligned on the vertical $z$-direction, with a module equal to $v_{c s t}$. As the mesh velocity is 449 identical for all mesh nodes, the mesh will keep unchanged all along the simulation. Note that for the 450 reference simulation, the geometry and its discretization are the same as for the moving ingot at initial 451 state. Nonetheless, the value of mesh velocity is null and by consequence, a fixed mesh will be used for 452 the whole simulation.

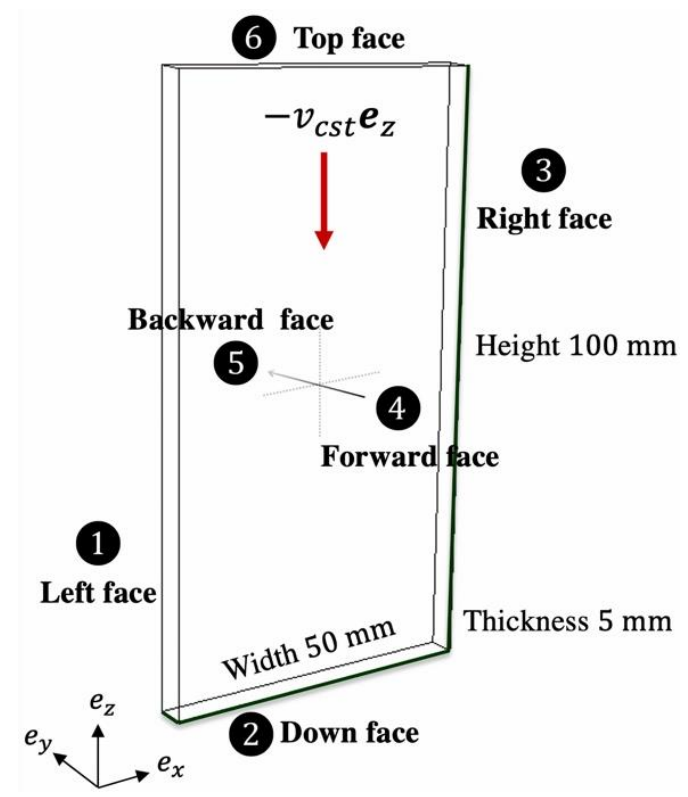

Fig.4. Geometry of test case simulation of the moving ingot under solidification

Indeed, this moving simulation could be seen as a representation of a fixed slice of the metal product for the CC process. Due to the symmetry of the system, only half of the slice is modeled. The six faces of the ingot are numbered in order to clearly describe the boundary conditions. Note that the left face, as well as the backward and forward faces, are defined as symmetry planes. In the following, the associated thermal and mechanical boundary conditions, for the moving ingot simulation, are summarized in Table 4. 


\begin{tabular}{l|c|c|c}
\hline $\begin{array}{c}\text { Face position } \\
\text { in Fig.4 }\end{array}$ & $\begin{array}{c}\text { Thermal boundary } \\
\text { conditions }\end{array}$ & \multicolumn{2}{|c}{ Mechanical boundary conditions } \\
\hline & & Fixed ingot (reference) & Moving ingot \\
\hline (1) Left & Adiabatic & Sliding $\left(\boldsymbol{v} \cdot \boldsymbol{e}_{x}=\mathbf{0}\right)$ & Sliding $\left(\boldsymbol{v} \cdot \boldsymbol{e}_{x}=0\right)$ \\
(2) Down & Flux by convection & Sticking $(\boldsymbol{v}=\mathbf{0})$ & Constant velocity $\left(\boldsymbol{v}=-v_{c s t} \boldsymbol{e}_{z}\right)$ \\
(3) Right & Flux by convection & Sticking $(\boldsymbol{v}=\mathbf{0})$ & Constant velocity $\left(\boldsymbol{v}=-v_{c s t} \boldsymbol{e}_{z}\right)$ \\
(4) Backward & Adiabatic & Sliding $\left(\boldsymbol{v} \cdot \boldsymbol{e}_{y}=0\right)$ & Sliding $\left(\boldsymbol{v} \cdot \boldsymbol{e}_{y}=0\right)$ \\
5 Forward & Adiabatic & Sliding $\left(\boldsymbol{v} \cdot \boldsymbol{e}_{y}=0\right)$ & Sliding $\left(\boldsymbol{v} \cdot \boldsymbol{e}_{y}=0\right)$ \\
6 Top & Adiabatic & Sticking $(\boldsymbol{v}=\mathbf{0})$ & Constant velocity $\left(\boldsymbol{v}=-v_{c s t} \boldsymbol{e}_{z}\right)$ \\
\hline
\end{tabular}

Table 4. Thermal and mechanical boundary conditions for the solidification test case

Heat is extracted from the metal through the right face and the bottom face using a convection-type expression for heat flux density, $q_{T}=h_{T}\left(T-T_{\text {ext }}\right)$, where the heat transfer coefficient $h_{T}$ and the external temperature $T_{\text {ext }}$ are constant. The metal is supposed to slide along the surrounding faces except at the top, bottom and left faces, on which a constant velocity $v_{c s t}$ is imposed in the z-direction $\boldsymbol{e}_{z}$. It should be reminded that the above-defined mechanical boundary condition is only applied in STEP II, as STEP I is over-simplified with solution $\boldsymbol{v}_{I}$ predefined, equal to $-v_{c s t} \boldsymbol{e}_{z}$, over the whole metal domain. Finally, note that for the reference simulation with fixed ingot, the energy boundary condition is the same as the above-detailed moving ingot simulation. For the mechanical boundary condition, sticking boundary condition is imposed on the top, bottom and left faces and all other faces are supposed to be purely sliding.

The initial temperature of the metal $T_{i n i}$ is chosen as the liquidus temperature $T_{L}$. Densities of the liquid and solid phases, $\langle\rho\rangle^{l}$ and $\langle\rho\rangle^{S}$, are taken as constant and equal to the value at $T_{L}, \rho_{L}$. Hence, solidification shrinkage is neglected and metal is assumed incompressible. Despite this assumption, in the momentum conservation equation relative to the liquid phase, the thermal convection in the liquid phase is taken into consideration by using the Boussinesq expression for the intrinsic liquid density: $\langle\rho\rangle^{l}=\rho_{L}\left(1-\beta^{l}\left(T-T_{L}\right)\right)$, where $\rho_{L}$ denotes the density of the liquid phase at the liquidus temperature and $\beta^{l}$ the thermal dilatation coefficient of the liquid phase. In the specific context of this verification test case, the solidification path is considered in an oversimplified form, assuming that the volume fraction of the solid phase evolves linearly with temperature in the solidification interval.

The values of process, numerical, and material parameters used in this first test are summarized in Table 5. Regarding material properties, they should be seen as representative of a carbon steel. As such, they are issued from different references and are simplified. For example, in this test, the thermal conductivity is assumed to be constant and equal for both the liquid and the solid phase. The latent heat of fusion is 

capacity relative to liquid and solid phases.

\begin{tabular}{llll}
\hline Material parameters & Symbol & Value & Unit \\
\hline Solidus temperature & $T_{S}$ & 1499 & ${ }^{\circ} \mathrm{C}$ \\
Liquidus temperature & $T_{L}$ & 1552 & ${ }^{\circ} \mathrm{C}$ \\
Densities & $\rho_{L},\langle\rho\rangle^{l},\langle\rho\rangle^{S}$ & 7060 & $\mathrm{~kg} \cdot \mathrm{m}^{-3}$ \\
Thermal dilatation coefficient of liquid & $\beta^{l}$ & $2.95 \times 10^{-4}$ & ${ }^{\circ} \mathrm{C}^{-1}$ \\
Secondary dendritic arm spacing & $\lambda_{2}$ & $5 \times 10^{-4}$ & $\mathrm{~m}$ \\
Liquid viscosity & $\mu^{l}$ & $4.2 \times 10^{-3}$ & $\mathrm{~Pa} \cdot \mathrm{s}$ \\
Solid specific heat & $\left\langle C_{p}\right\rangle^{s}$ & 400 & $\mathrm{~J} \cdot \mathrm{kg}^{-1} \cdot{ }^{\circ} \mathrm{C}^{-1}$ \\
Liquid specific heat & $\left\langle C_{p}\right\rangle^{l}$ & 800 & $\mathrm{~J} \cdot \mathrm{kg}^{-1} \cdot{ }^{\circ} \mathrm{C}^{-1}$ \\
Thermal conductivity for solid and liquid phases & $\langle k\rangle^{l},\langle k\rangle^{S}$ & 30 & $\mathrm{~W} \cdot \mathrm{m}^{-1} \cdot{ }^{\circ} \mathrm{C}^{-1}$ \\
Latent heat of fusion & $L_{f}$ & $3.09 \times 10^{5}$ & $\mathrm{~J} \cdot \mathrm{kg}^{-1}$ \\
\hline Process and numerical parameters & & & \\
\hline Initial temperature & $T_{i n i}$ & 1552 & ${ }^{\circ} \mathrm{C}$ \\
External temperature & $T_{e x t}$ & 25 & ${ }^{\circ} \mathrm{C}$ \\
Heat transfer coefficient & $h_{T}$ & 1000 & $\mathrm{~W} \cdot \mathrm{m}^{-2} \cdot{ }^{\circ} \mathrm{C}^{-1}$ \\
Downward moving velocity & $v_{c s t}$ & 0.016167 & $\mathrm{~m} \cdot \mathrm{s}^{-1}$ \\
Time step & $\Delta t$ & 0.1 & $\mathrm{~s}$ \\
\hline
\end{tabular}

Table 5. Values of material and numerical parameters used for the verification test case.

\subsubsection{Results}

Fig. 5 illustrates the progress of the ingot solidification at time $t=40 \mathrm{~s}$. In Fig. 5a, results on the front symmetry plane are shown, comparing the reference static ingot simulation (left half part of the figure) and the moving ingot simulation (right half part). More precisely, the left part of Fig. 5a illustrates the temperature field, $T$, and the fluid flow, $\boldsymbol{v}_{I I}$, in the reference static ingot simulation. Note that the velocity field can also be considered as $\boldsymbol{v}_{I I}-\boldsymbol{v}_{m s h}$, as $\boldsymbol{v}_{m s h}$ is indeed null in such a pure Eulerian framework simulation. The right part of Fig. 5a describes the temperature field, $T$, and the relative fluid

492 flow, $\boldsymbol{v}_{I I}-\boldsymbol{v}_{m s h}$, for the moving ingot simulation in which $\boldsymbol{v}_{m s h}=-\boldsymbol{v}_{c s t} \boldsymbol{e}_{z}$ is imposed to the whole 493 computational mesh. It should be mentioned that the spatial positions of the two ingots are indeed 494 different, i.e. with a relative vertical distance difference. The two simulation results (separated by the 495 dotted black symmetric line) are put side by side in order to facilitate comparison.

496 It can be seen that, by eliminating the mesh velocity $\boldsymbol{v}_{m s h}$, the same velocity field is found, which 497 corresponds in both simulations to the velocity induced by the thermal convection in the liquid phase.

498 Moreover, an identical temperature field is recovered in the moving ingot simulation compared to the 499 reference static ingot simulation. The liquidus and solidus isotherms, highlighted with white lines, are 
perfectly connected on the (yz) symmetry plane. It is worth noting that in the moving ingot simulation, energy transport relative to the solid phase is taken into consideration. The relative movement between the solid and liquid phases is also considered. As shown in Fig. 5b, the velocity field $\boldsymbol{v}_{I I}$, in the moving simulation, is a mixture of the downward moving velocity and the thermal convection in the liquid phase. It thus differs from the constant downward velocity, $\boldsymbol{v}_{I}$. Finally, note that as shown in Fig. 5a, the amplitude of the fluid flow induced by convection is smaller than that of the constant downward movement. This just fits in the fact for the presence of a global downward fluid flow is in Fig. $\mathbf{5 b}$.
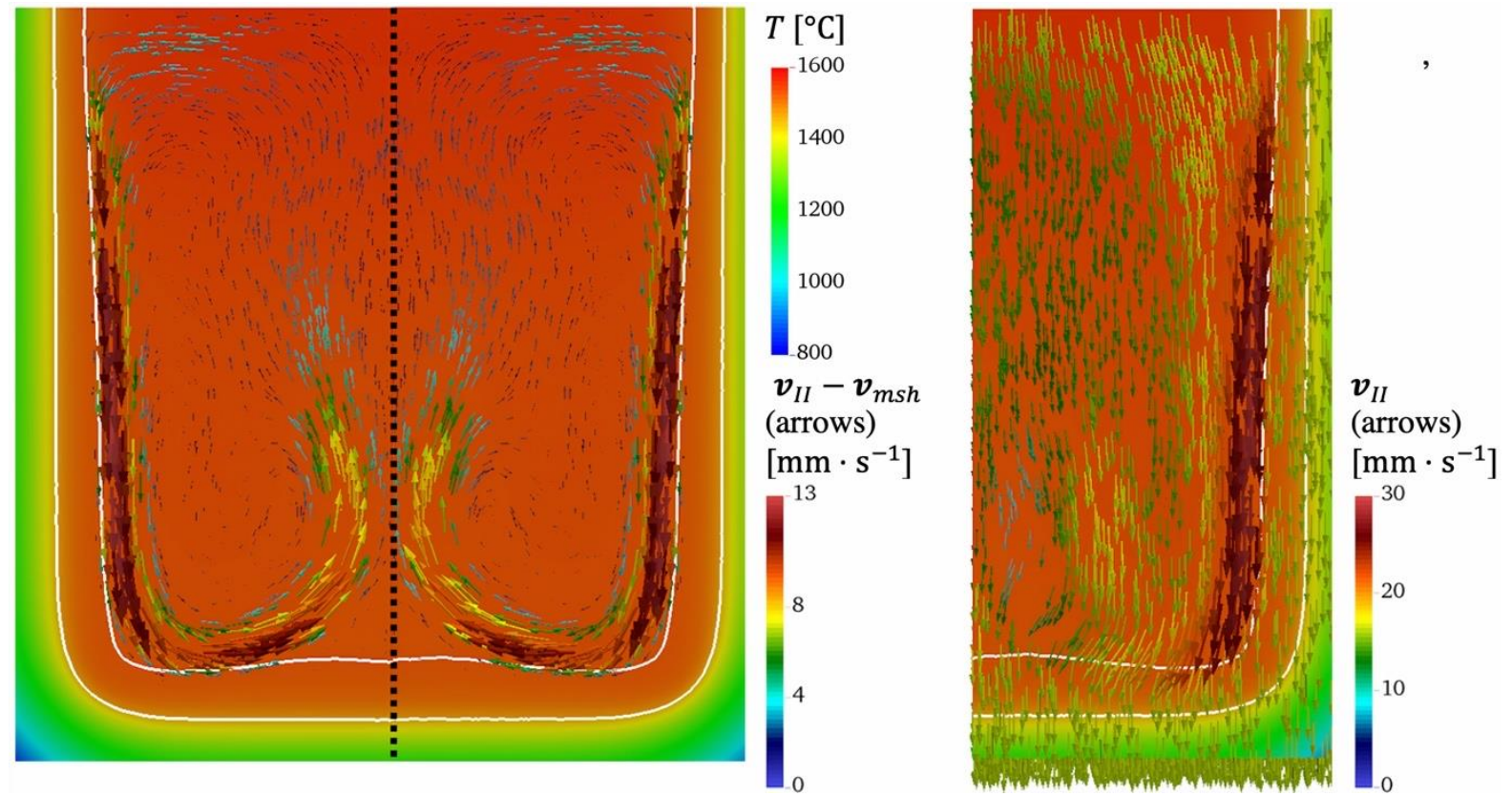

Fig. 5. Snapshot of solidification simulation showing (a) a comparison of the temperature field, $T$, and the relative fluid flow, $\boldsymbol{v}_{I I}-\boldsymbol{v}_{m s h}$, at time $t=40 \mathrm{~s}$, respectively in the reference static ingot simulation (left half part) and in the moving ingot simulation (right half part); (b) the velocity field $\boldsymbol{v}_{I I}$ associated with fluid flow in the moving ingot simulation. Liquidus and solidus isotherms are highlighted with white lines.

\subsection{Application to continuous casting}

509 In this section, the application of the proposed two-step formulation to the simulation of an industrial 510 pilot CC process is proposed. This constitutes a preliminary step, for the present algorithm, before 511 addressing large and complex industrial CC processes. In particular, it intends to demonstrate its capability to compute concurrently, in a quasi-industrial configuration, fluid flow and stress-strain in the solid shell, in the mold region as well as in the secondary cooling zone.

\subsubsection{Model description}

516 The initial configuration of the model is given in Fig. 6a, with the consideration of a submerged entry 517 nozzle and the gas sub-domain. The cast product is a slab of thickness $100 \mathrm{~mm}$ and width $750 \mathrm{~mm}$, the 518 primary cooling being operated by a mold of height $800 \mathrm{~mm}$. Heat is extracted from the metal through 519 the lateral vertical surfaces (with normal $\boldsymbol{e}_{x}$ and $\boldsymbol{e}_{y}$ ). Constant flux is imposed along surfaces in contact 
with the mold and a convection type expression is defined for flux associated with free surfaces located

521 below the mold exit in order to consider water cooling effect. It should be noticed that a small insulation

522 zone $(50 \mathrm{~mm})$ is imposed in the vicinity of the meniscus so as to always maintain a liquid-gas interface

523 in contact with the mold. The metal is supposed to slide vertically along the vertical surfaces. As

524 mentioned previously, although the mold is present in Fig. 6a, it is actually not explicitly modeled in

525 the simulation.

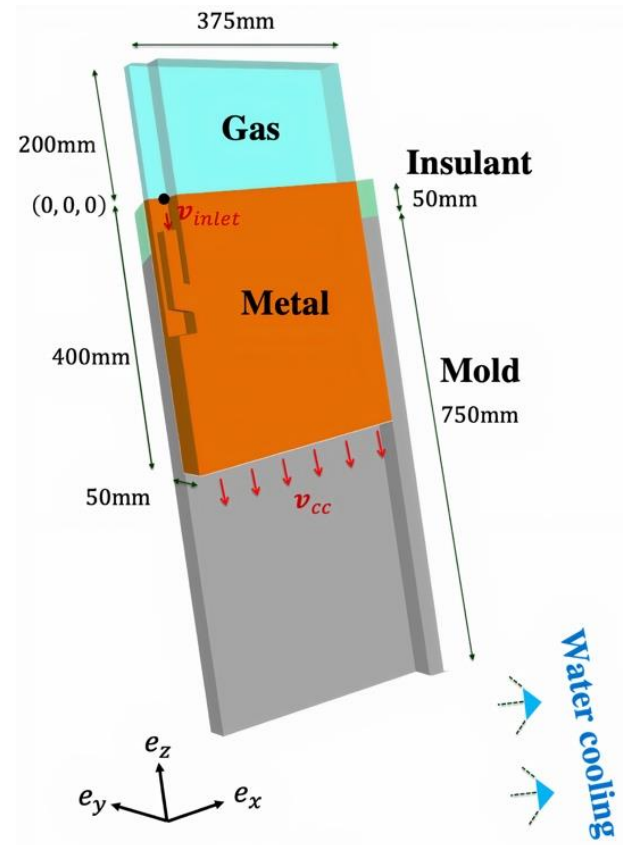

(a)
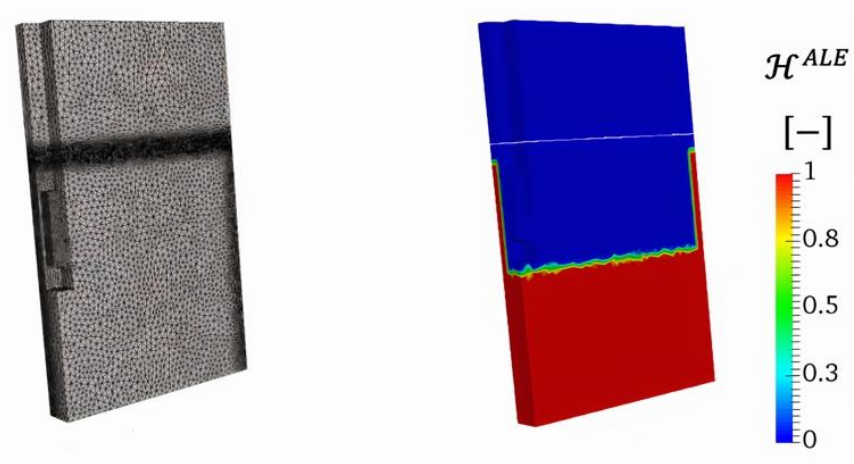

(b) (c)

Fig. 6. Representation of (a) a schematic illustration of the 3D CC model at its initial configuration; (b) the associated initial mesh and (c) the constant Heaviside function, $\mathcal{H}^{A L E}$, defined in this simulation, assuring a smooth transition of mesh velocity between ALE and Eulerian zones.

A free velocity is considered on the upper surface of the simulation domain, allowing gas to flow in and out freely. The surrounding faces of the entry nozzle are defined as sticking. The bottom surface of the metal sub-domain is imposed with the constant vertical casting velocity $\boldsymbol{v}_{c c}$. Moreover, another constant vertical velocity $\boldsymbol{v}_{\text {inlet }}$ is imposed at the top surface of the entry nozzle, modeling the entry of the liquid from the tundish to the mold region. The same mechanical boundary conditions as described above are applied to both STEP I and STEP II, except for the surrounding vertical surfaces in contact with the mold in STEP II. Along these surfaces, sticking boundary conditions are assumed, in order to reduce numerical instabilities due to the presence of the intensive nozzle jet in contact with the mold.

534 Only a quarter of the full geometry is modeled considering the symmetry of the system. The initial mesh

535 is defined in Fig. 6b. Isotropic mesh with a constant mesh size of $10 \mathrm{~mm}$ is used over both the metal 536 and gas sub-domains except for two critical regions. First, in the $20 \mathrm{~mm}$ thick metal/gas transition zone, 537 the mesh is refined in an anisotropic manner with a mesh size of $1.5 \mathrm{~mm}$ in the vertical direction and 10 $538 \mathrm{~mm}$ in the horizontal directions. An extra $20 \mathrm{~mm}$ thick transition zone is defined on each side of the 539 transition zone. This is done in order to ensure a smooth evolution of the mesh size between the transition 540 zone and the regions with isotropic coarse mesh size, thus reducing possible numerical instabilities due 
541 to poor mesh quality in this critical region around the metal/gas boundary. Besides, for the metallic zone 542 in the vicinity of the mold, isotropic refined mesh is used, with a minimum mesh size of $4 \mathrm{~mm}$, to ensure 543 a reliable stress-strain analysis in the very thin solid shell (on average, several centimeters thick in the 544 mold region). The number of mesh elements in the initial mesh as defined in Fig. $6 \mathbf{b}$ is about 640000. 545 It is worth noting that the number of finite elements will keep increasing with a globally linear evolution 546 over time.

547 The related constant Heaviside function, $\mathcal{H}^{A L E}$, as introduced previously in Section 2.2, is shown in Fig. 548 6c. It is smoothed over a total thickness of $20 \mathrm{~mm}$ around the zero-isovalue, i.e. the green-colored line, 549 describing the boundary line between the ALE and Eulerian zones. Remind that it ensures the smooth 550 transition of the mesh velocity between these two zones. More precisely, the explicit definition of the 551 mesh velocity in the mushy and solid zones being given by Eq. (20), the mesh velocity over the whole 552 simulation domain writes $\boldsymbol{v}_{m s h}=\mathcal{H}^{A L E}\left(g^{l} \boldsymbol{v}_{c c}+g^{S} \boldsymbol{v}_{I}\right)$.

553 The material properties are those of a 40CrMnMoS8-6 steel grade, as already documented in a previous 554 paper, ${ }^{[15]}$ with several additional simplifications detailed below. Firstly, constant and equal densities are 555 taken for liquid and solid phases, $\langle\rho\rangle^{l}=\langle\rho\rangle^{s}$ except in the momentum conservation equation of STEP 556 II where the density of the liquid phase follows the Boussinesq approximation, $\langle\rho\rangle^{l}=\rho_{L}(1-$ $\left.557 \beta^{l}\left(T-T_{L}\right)\right)$, in order to model the thermal convection of the liquid phase. Besides, dilatation of the 558 solid phase is taken into consideration in the mass conservation equation of STEP I, such as $\langle\rho\rangle^{S}=$ $559 \rho_{S}\left(1-\beta^{S}\left(T-T_{S}\right)\right)$ to allow a stress-strain analysis for the solid phase. Secondly, constant specific 560 heat is assumed for both solid and liquid phases. The latent heat of fusion is also taken as constant. Note 561 that the solidification path as well as the mechanical properties relative to the constitutive laws of solid 562 are kept as the same as in reference [15]. Finally, values of the material properties used together with 563 simulation parameters are summarized in Table 6. It should be mentioned that properties of the gas are 564 oversimplified in the current simulation in order to reduce possible numerical instabilities at the $565 \mathrm{metal} /$ gas boundary. Nonetheless, these simplifications have almost no effect in the main focus of this 566 simulation, i.e. the concurrent fluid flow and stress-strain analyses in the metal sub-domain. 


\begin{tabular}{|c|c|c|c|}
\hline Material parameters & Symbol & Value & Unit \\
\hline Solidus temperature & $T_{S}$ & 1431 & ${ }^{\circ} \mathrm{C}$ \\
\hline Liquidus temperature & $T_{L}$ & 1490.5 & ${ }^{\circ} \mathrm{C}$ \\
\hline Specific heat of solid and liquid & $\left\langle C_{p}\right\rangle^{l},\left\langle C_{p}\right\rangle^{s}$ & 800 & $\mathrm{~J} \cdot \mathrm{kg}^{-1} \cdot{ }^{\circ} \mathrm{C}^{-1}$ \\
\hline Specific heat of gas & $C_{p}^{G}$ & 1000 & $\mathrm{~J} \cdot \mathrm{kg}^{-1} \cdot{ }^{\circ} \mathrm{C}^{-1}$ \\
\hline Latent heat of fusion & $L_{f}$ & $2.4 \times 10^{5}$ & $\mathrm{~J} \cdot \mathrm{kg}^{-1}$ \\
\hline Metal density & $\rho_{L}, \rho_{S},\langle\rho\rangle^{l},\langle\rho\rangle^{S}$ & 7000 & $\mathrm{~kg} \cdot \mathrm{m}^{-3}$ \\
\hline Gas density & $\rho^{G}$ & 6000 & $\mathrm{~kg} \cdot \mathrm{m}^{-3}$ \\
\hline Thermal dilatation coefficient of liquid & $\beta^{l}$ & $1 \times 10^{-4}$ & ${ }^{\circ} \mathrm{C}^{-1}$ \\
\hline Thermal dilatation coefficient of solid & $\beta^{s}$ & $1 \times 10^{-5}$ & ${ }^{\circ} \mathrm{C}^{-1}$ \\
\hline Secondary dendrite arm spacing & $\lambda_{2}$ & $5 \times 10^{-4}$ & $\mathrm{~m}$ \\
\hline STEP I | STEP II & $\mu^{G}$ & $1 \mid 1 \times 10^{-1}$ & $\mathrm{~Pa} \cdot \mathrm{s}$ \\
\hline STEP I | STEP II & $\mu^{l}$ & $1 \mid 5 \times 10^{-3}$ & $\mathrm{~Pa} \cdot \mathrm{s}$ \\
\hline Thermal conductivity in liquid & $\langle k\rangle^{l}$ & 30 & $\mathrm{~W} \cdot \mathrm{m}^{-1} \cdot{ }^{\circ} \mathrm{C}^{-1}$ \\
\hline Thermal conductivity in solid & $\langle k\rangle^{s}$ & 30 & $\mathrm{~W} \cdot \mathrm{m}^{-1} \cdot{ }^{\circ} \mathrm{C}^{-1}$ \\
\hline Thermal conductivity in gas & $k^{G}$ & 0.1 & $\mathrm{~W} \cdot \mathrm{m}^{-1} \cdot{ }^{\circ} \mathrm{C}^{-1}$ \\
\hline Simulation parameters & Symbol & Value & Unit \\
\hline Casting temperature & & 1520.5 & ${ }^{\circ} \mathrm{C}$ \\
\hline External temperature & $T_{\text {ext }}$ & 25 & ${ }^{\circ} \mathrm{C}$ \\
\hline Constant flux at narrow face & & $8 \times 10^{5}$ & $\mathrm{~W} \cdot \mathrm{m}^{-2}$ \\
\hline Constant flux at wide face & & $1.2 \times 10^{6}$ & $\mathrm{~W} \cdot \mathrm{m}^{-2}$ \\
\hline Heat transfer coefficient at narrow face & & 1100 & $\mathrm{~W} \cdot \mathrm{m}^{-2} \cdot{ }^{\circ} \mathrm{C}^{-1}$ \\
\hline Heat transfer coefficient at wide face & & 150 & $\mathrm{~W} \cdot \mathrm{m}^{-2} \cdot{ }^{\circ} \mathrm{C}^{-1}$ \\
\hline Time step & $\Delta t$ & 0.1 & $\mathrm{~s}$ \\
\hline Casting velocity in the vertical direction & $\boldsymbol{v}_{c c} \cdot \boldsymbol{e}_{z}$ & -0.015 & $\mathrm{~m} \cdot \mathrm{s}^{-1}$ \\
\hline Liquid inlet velocity in the vertical direction & $\boldsymbol{v}_{\text {inlet }} \cdot \boldsymbol{e}_{z}$ & -0.88 & $\mathrm{~m} \cdot \mathrm{s}^{-1}$ \\
\hline
\end{tabular}

Table 6. Simulation and material parameters in the 3D CC test case. Non-listed material properties can be found in Reference [15].

\section{$568 \quad$ 3.2.2 Results}

569 Fig. 7 illustrates the non-steady regime of the simulation through the distribution of solid fraction, 570 velocity vectors and temperature, respectively at times $t=10 \mathrm{~s}$ and $t=30 \mathrm{~s}$. The metal/gas boundary 571 is represented by the green surface. As shown in Fig. 7a, after $10 \mathrm{~s}$ of solidification process, a solid shell 572 has already formed along both the narrow and wide cooling faces. However, it is worth noting that on 573 the narrow face, an unsolidified area is present with a slender oval shape (position A). The formation 574 mechanism of this particular area is indeed a conjugate effect of the initial modeling configuration (the 575 simulation is started with a metal sub-domain in fully liquid state) and of the impact from the hot nozzle 
jet onto the mold region. More precisely, as shown in Fig. 7b, at time $10 \mathrm{~s}$, a main intense flow can be observed in the mold region due to the injected flow from the nozzle entry. This nozzle jet is split into two vortices when it meets the narrow face, with one in the upward direction and another in the downward direction. As it can be seen by comparing Fig. 7a and Fig. 7b, the unsolidified area labeled A is located at the impingement zone of the main fluid flow on the narrow face. By consequence, starting from a fully liquid state, this hot jet impingement delays the formation of the mushy zone, as well as the solid shell, on this particular area, at an early stage of the solidification process. The temperature field, at this time $t=10 \mathrm{~s}$, is shown in Fig. 7c. It can be seen that there is an impact of the fluid flow on the solidification process. Especially on the narrow face, the temperature of the impingement zone remains hottest, compared to its neighboring areas, with consequence on the distribution of the solid on the narrow face already previously commented.

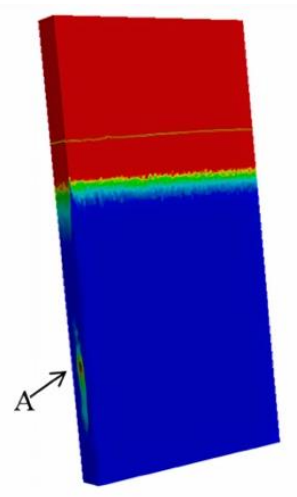

(a) $10 \mathrm{~s}$

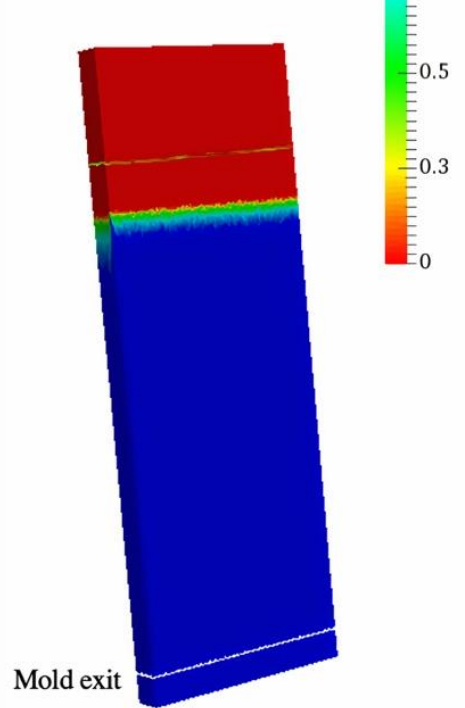

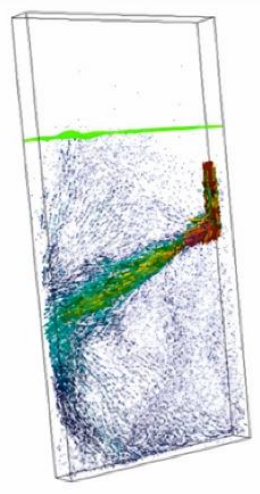

(b) $10 \mathrm{~s}$

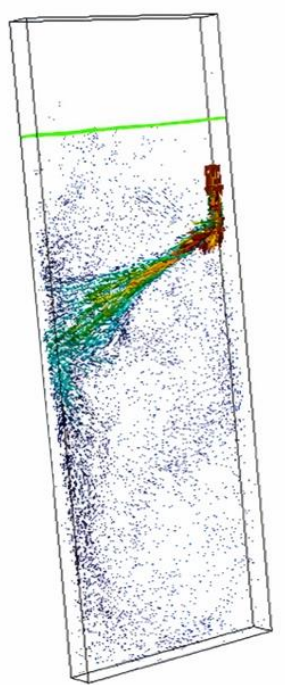

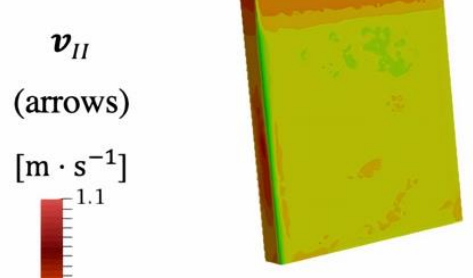

(c) $10 \mathrm{~s}$

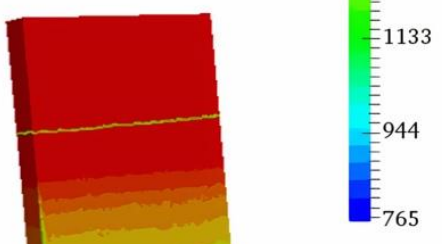

Fig. 7. Results for $g^{s}, \boldsymbol{v}_{I I}$ and $T$ respectively at time $t=10 \mathrm{~s}$ with (a) the distribution of solid fraction along the domain boundary, (b) the velocity vectors throughout the domain, (c) the temperature field along the product surface. The same fields at time $t=30 \mathrm{~s}$ are shown on the bottom line: figures (d) to (f).

587 Fig. 7d shows the solid fraction at time $t=30 \mathrm{~s}$, when the metal sub-domain has just exited the mold 588 region (the mold exit is represented by the white line). It can be seen that the unsolidified area labeled 589 A in Fig. 7a has disappeared. This expresses the fact that the casting conditions are sustainable, without 
major risk of liquid leakage at the exit of the mold. However, it is worth noting that, as shown in Fig.

$5917 \mathbf{7 e}$, the direction and magnitude of the main fluid flow in the mold region are nearly the same as that at

592 time $t=10 \mathrm{~s}$. By consequence, the impingement area keeps being located approximately at position A.

593 Although the unsolidified area is no more present on the narrow cooling face at time $t=30 \mathrm{~s}$, the impact

594 of the fluid flow remains significant, and a permanent stabilized hot spot exists. Fig. 7f shows the

595 temperature field at time $t=30 \mathrm{~s}$. It can be seen that the solid shell in the impingement area, is hotter

596 than in the surrounding area.

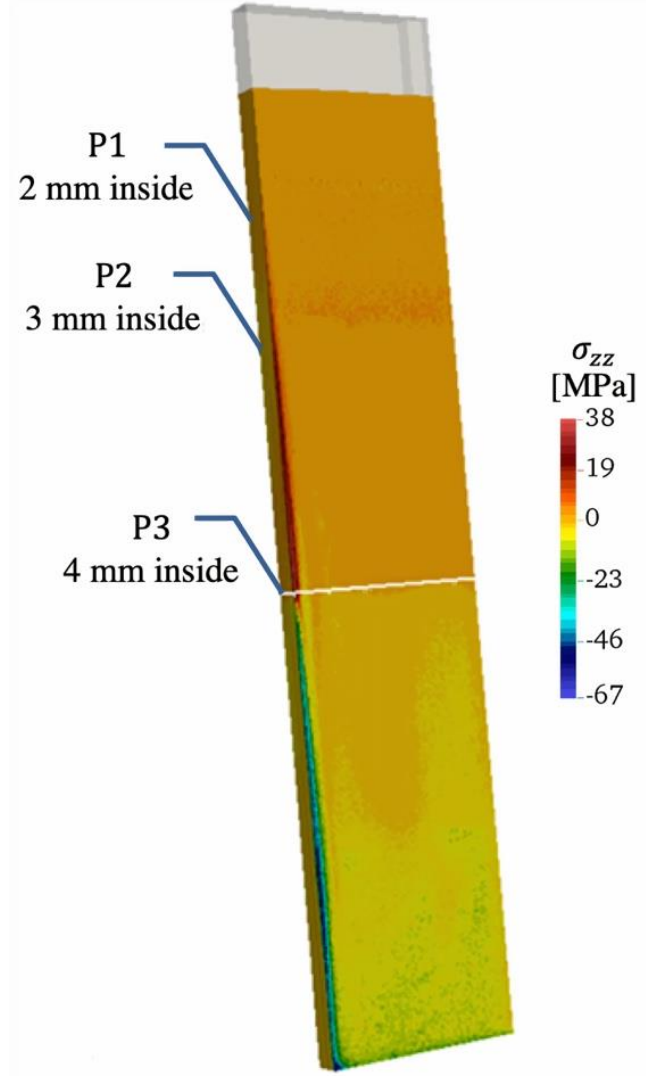

(a)

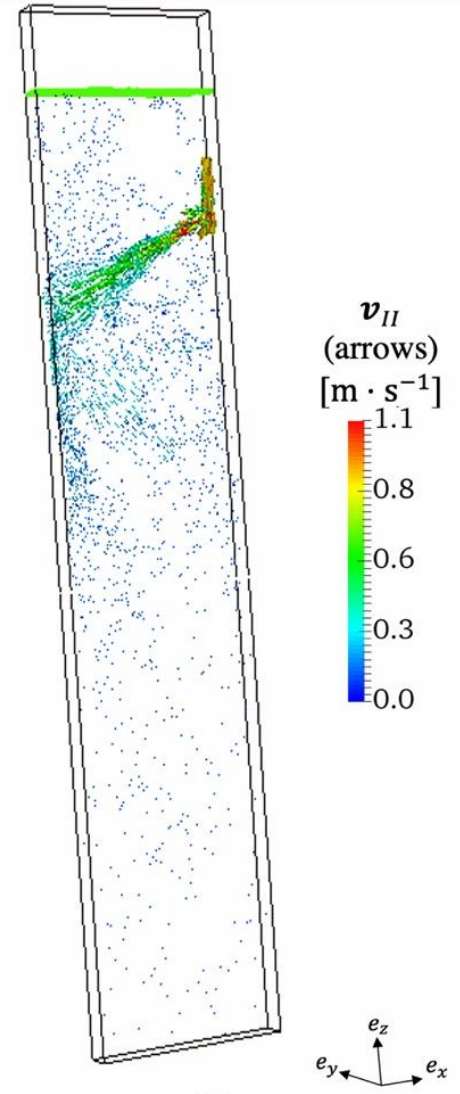

(b)

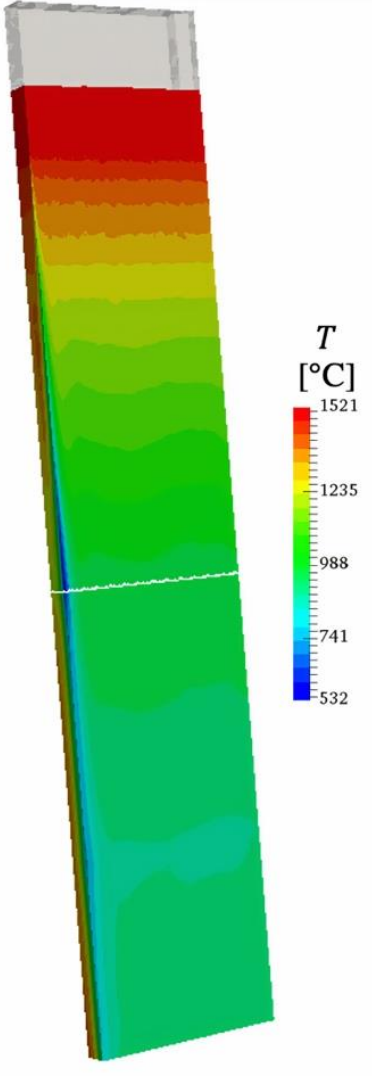

(c)

Fig. 8. Results at time $t=80 \mathrm{~s}$, showing (a) $\sigma_{z z}$, the longitudinal stress along the cooling faces with the white line representing the mold exit; (b) The fluid flow (arrows, $\boldsymbol{v}_{I I}$ ) with the presence of the nozzle jet; (c) The temperature field along the cooling faces.

Fig. 8 illustrates the results corresponding to time $t=80 \mathrm{~s}$. The longitudinal (vertical) stress component $\sigma_{z z}$ along the external cooling faces of the product is shown in Fig. 8a, revealing that the solid shell is under traction within the mold region, in particular along the edge, while in compression after the mold exit, with maximum values reaching respectively $38 \mathrm{MPa}$ and $-67 \mathrm{MPa}$. The fluid flow is concurrently computed, represented by colored arrows in Fig. 8b. The length and color of arrows are related to the vector magnitude. It can be seen that the fluid flow is intensive in the mold region and slows down when reaching the mold exit. Fig. 8c shows the temperature field over the two cooling faces. Because heat extraction in secondary cooling is much lower than in the mold region, a minimum of temperature along the edge is found at the mold exit (here $532{ }^{\circ} \mathrm{C}$ ). It is worth noting that the extra-cooling along the edge of the product is indeed overestimated with respect to the real industrial process. This is because the 
formation of an air gap between the product and the mold is not under consideration in the present model.

608 The presence of such an air gap has an important influence on heat extraction in the vicinity of the corner,

609 i.e. slowing down the cooling process and thus resulting in higher values of temperature in this region 610 than calculated here. ${ }^{[26]}$

611 It is then interesting to investigate when the steady-state regime is reached in the present non-steady 612 simulation. For this specific purpose, three fixed observation points are defined at positions $(x, y, z)=$ $613(373,0,-200),(372,0,-400)$ and $(371,0,-800)$ with units in $\mathrm{mm}$. They are respectively denoted as P1, 614 P2 and P3 and their locations can be seen in Fig. 8a. Remind that the $(0,0,0)$ position is located at the $615 \mathrm{metal} /$ gas nominal interface, at the intersection between the two vertical symmetry planes, as shown in 616 Fig. 6a (black dot). All three fixed positions are in the mold region, in the vicinity of the middle of the 617 narrow cooling face. Position P3 is located exactly in the transversal cross section at the mold exit, 4 $618 \mathrm{~mm}$ deep in the thickness of the solid shell. Calculated evolutions of the temperature and the von Mises 619 stress at these predefined positions are recorded during $60 \mathrm{~s}$ and plotted in Fig. 9.

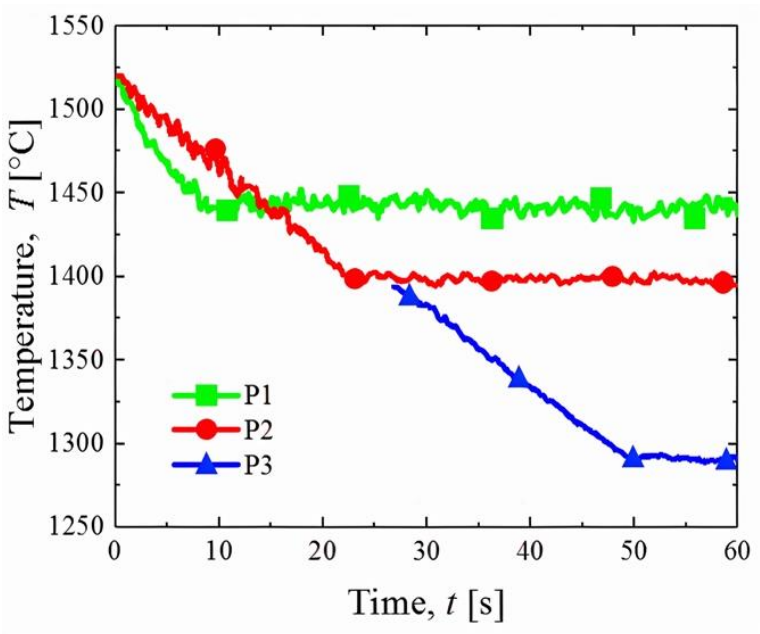

(a)

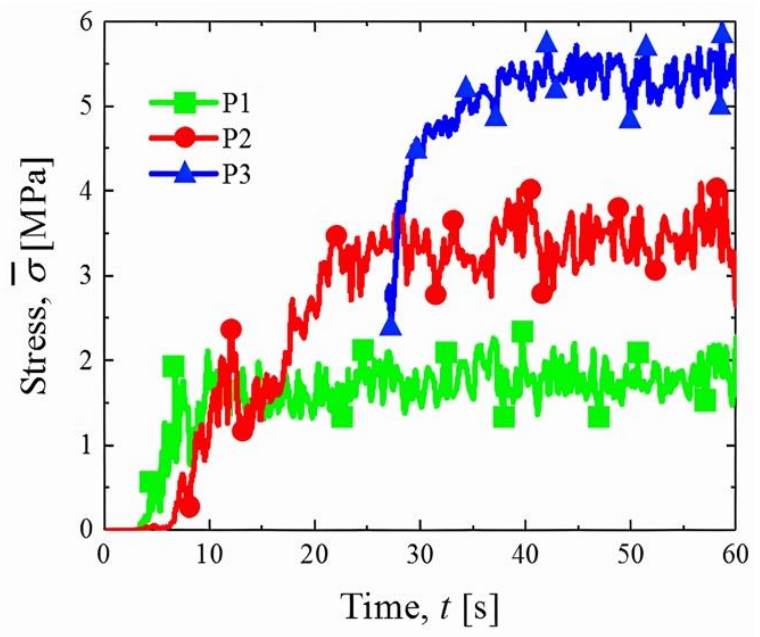

(b)

Fig. 9. Evolution of temperature $T$ (left), and von Mises stress $\bar{\sigma}$ (right) at three fixed locations P1, P2 and P3, defined in Fig. 8a.

620 It can be noted that for position P3, results are available only after about $25 \mathrm{~s}$ of simulation, as shown

621 by the blue colored lines in Fig. 9. This is because position P3 is outside the initial simulation domain, 622 as defined in Fig. 6a: it is then reached after a certain process time $\Delta z / v_{c c}=400 / 15=26.7 \mathrm{~s}$ in the 623 present non-steady state approach. Conversely, the other two positions are located at the initial metal 624 sub-domain and thus results are available from the beginning.

625 It can be seen that the steady-state regime is reached within the entire mold region after about $50 \mathrm{~s}$, 626 either from the thermal point of view (Fig. 9a) or from the mechanical point of view (Fig. 9b). As P3 is 627 the lower position in the mold it is also the last one to stabilize. After this position has been attained by 628 the growing computational mesh, it can be observed that it requires about $23 \mathrm{~s}$ to reach a steady state, 629 which is indeed a short process time, corresponding only to a casting length of $345 \mathrm{~mm}$. Let us note 630 however that reaching the steady state would be longer if the heat transfer within the mold would be 
631 considered in the simulation (instead of a constant imposed heat flux here). It is also interesting to note 632 that the mechanical steady-state regime is reached almost at the same time as the thermal steady-state 633 regime, for all three points. This is coherent with the boundary conditions prescribed along the cooling 634 faces, where only vertical displacement is allowed and the cooling faces cannot separate from the mold. 635 Thus, under this assumption, there is no coupling feedback from the mechanical simulation toward the 636 thermal simulation. Finally, one observes that steady state is in fact not perfect, all evolution curves 637 being affected by some perturbations. These perturbations are indeed inherent to the non-steady state 638 simulation. Actually, during the simulation, the mobile mesh passes by the different fixed positions. The 639 calculated value at a certain process time and for a selected position depends on the local spatial 640 interpolation which can be done at that precise moment. These fluctuations tend to increase with the 641 local mesh size, and with the local gradient of the interpolated solution fields. This is why fluctuations 642 are more visible for stress evolution (high gradients, see also hereunder) than for temperature evolution 643 (lower gradients).

644 Fig. 10a illustrates the solid fraction and the stress component in $z$-direction $\left(\sigma_{z z}\right)$ over the cross section 645 at mold exit, at time $=80 \mathrm{~s}$, after the steady-state regime is achieved in the whole mold region.

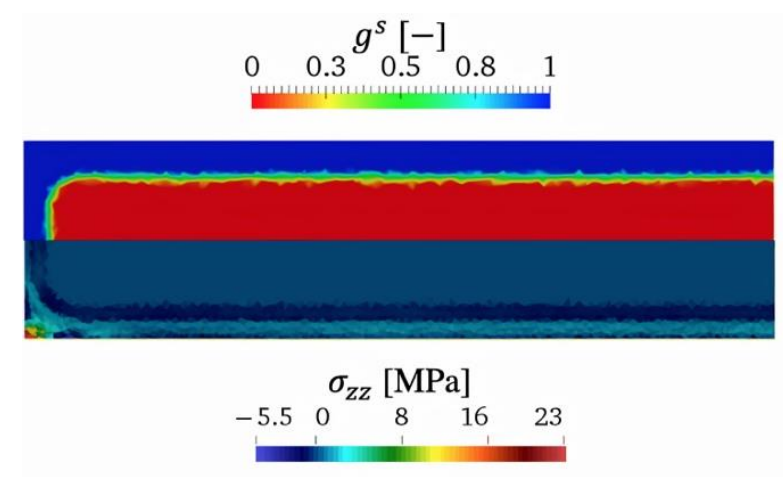

(a)

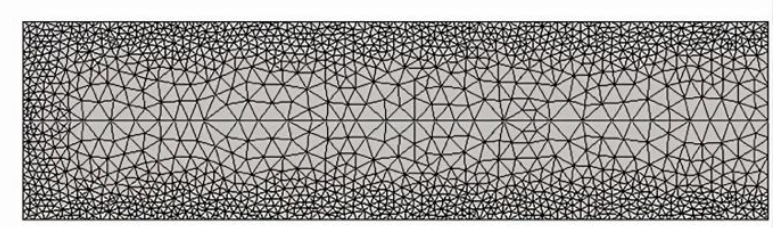

(b)

Fig. 10. (a) distribution of solid fraction $g^{s}$ (top half of the figure), and of the stress component in the $z$ direction $\sigma_{z z}$ (bottom half of the figure) at time $t=80 \mathrm{~s}$ in the transverse cross-section at mold exit; (b) section of the mesh over this cross-section in a $2 \mathrm{D}$ configuration.

646 First, it can be seen that a solid shell with thickness about $15 \mathrm{~mm}$ is formed on the wide cooling face. 647 Given the intensity of the extracted fluxes, of the order of $1 \mathrm{MW} \mathrm{m}^{-2}$, this result is as expected. It 648 compares well, for instance, with experimental measurements published by Thomas et al.., ${ }^{[28]}$ and with 649 associated numerical simulations performed by Pfeiler et al., ${ }^{[29]}$ based on similar casting conditions. The 650 slab format of the pilot machine studied here having a thin thickness, approximately one third of the 651 product is solidified at mold exit. It can also be seen that the corner of the cooling faces is submitted to 652 a high tensile stress, with a maximum value reaching $23 \mathrm{MPa}$, whereas the solidification front undergoes 653 smaller compression stress. Nonetheless, it should be mentioned that this maximum predicted value 654 might be unrealistic as the cooling faces are highly constrained (the velocity along faces is imposed to 655 be vertical). Finally, note that a simple remeshing strategy is adopted to ensure a correct implementation 656 of the above stress-strain analysis in such thin solid shell. Fig. 10b illustrates the corresponding 657 equivalent mesh over the whole cross-section. It is worth noting that the exact presentation of the mesh 
in such a cross-section of the $3 \mathrm{D}$ geometry would be unintelligible. This is why an equivalent 2D mesh has been drawn, with local size corresponding to the 3D mesh size. Being aware of this simplification,

660 it can be seen that the mesh is refined close to the cooling faces, providing a sufficient number of 661 elements to describe the stress gradient through the thickness of the solid shell.

662 In order to get a clearer insight of the stress developed in the solid shell, Fig. 11 illustrates the stress 663 profiles, $\sigma_{z z}$, over the half-thickness of the product, at three different locations in the $(y z)$ symmetry 664 plane. These profiles are then perpendicular to the wide face, respectively at $200 \mathrm{~mm}$ above the mold 665 exit (green line, square symbols), at the mold exit (red line, dots) and $200 \mathrm{~mm}$ below the mold exit (blue 666 line, triangles). The $y$ coordinate varies from 0 (central core of the product) to $50 \mathrm{~mm}$ (center of the 667 wide cooling face). The associated solidus position of each profile is represented by the dotted lines as 668 shown in Fig. 11.

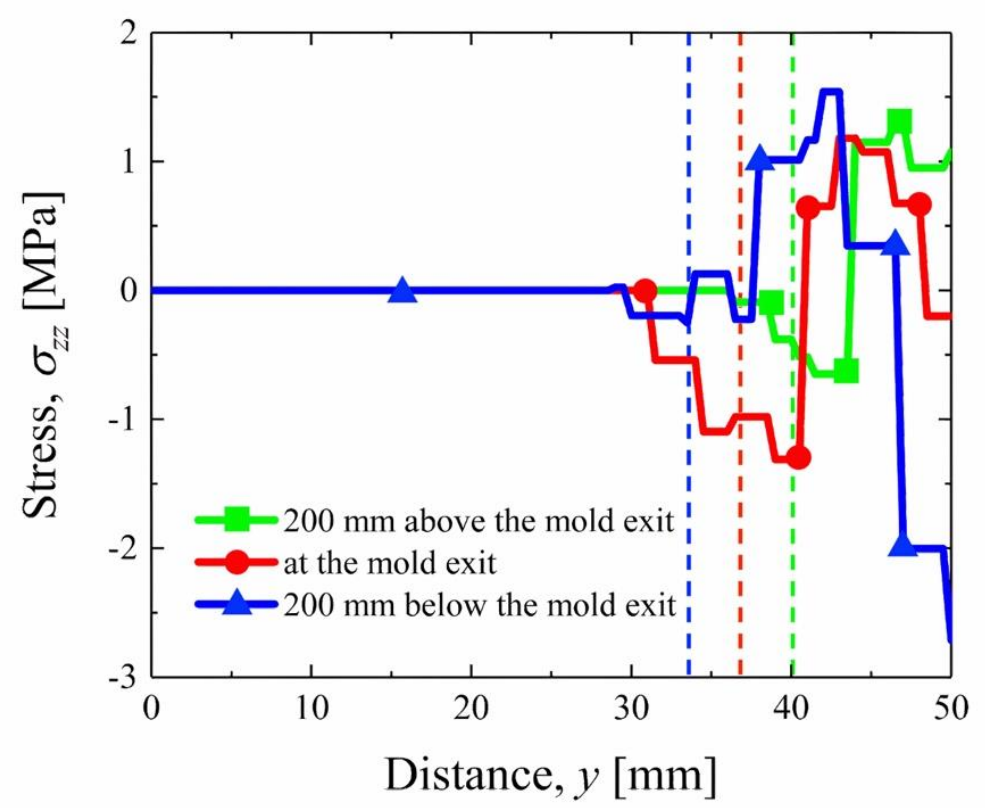

Fig. 11. $\sigma_{z z}$ stress profile through the thickness in the transverse symmetry plane at time $80 \mathrm{~s}$, respectively at three different locations, $200 \mathrm{~mm}$ above the mold exit, at the mold exit and $200 \mathrm{~mm}$ below the mold exit. The dotted lines represent locations of the solidus, associated with each stress profile.

669 It can be seen that above the mold exit, the vertical stress along the wide cooling face (at $y=50 \mathrm{~mm}$ in

670 Fig. 11) is tensile. Nonetheless, about half of the solid shell near the cooling face is still under compression, expressing the necessary mechanical equilibrium along the vertical direction. At the mold

672 exit, the vertical stress on the wide cooling face starts to shift from tensile to slightly compressive, while

673 the vertical stress at the solidification front is still compressive. Below the mold exit, the vertical stress

674 along the wide cooling face becomes fully compressive, and by consequence, a global tensile stress is 675 obtained deeper in the solid shell. It is worth noting that for both three locations, the part of the mushy 676 zone with higher solid fractions, close to the solidus, is always submitted to a vertical compressive stress.

677 Finally, as expected, all vertical stresses recover to zero when they reach the fully liquid regions. Note 
that both the evolution of the stress profile along the vertical direction and the stress level are in agreement with other simulation results obtained by either Risso et al., ${ }^{[30]}$ or Zappulla et al., ${ }^{[31]}$ for

680 similar slab casting configurations.

\section{Conclusions}

683 A new partitioned solution algorithm has been developed to simulate steel CC processes, as an extension

684 of a former two-step algorithm initially developed for ingot casting application. ${ }^{[15]}$ This new algorithm

685 allows a concurrent computation of fluid flow in the liquid and mushy regions, and stress-strain in the

686 solid, in the context of a non-steady state simulation of CC processes. The algorithm consists of a

687 partitioned solution strategy, to calculate, at each time increment, a solid-oriented solution, a fluid-

688 oriented solution, and a coupled thermal solution. The three specific features of this new solution

689 algorithm, which have been specially developed to simulate $\mathrm{CC}$ processes, are the followings:

690 - A global non-steady-state approach allows simulating the transient regime up to the convergence to 691 the steady state regime. For this, the computational mesh continuously moves and grows, in accordance with the imposed extraction speed.

- This evolving mesh is partitioned in three regions: the solid shell, which is treated as a pure Lagrangian zone; the liquid nozzle region as a pure Eulerian zone; and an intermediate EulerianLagrangian zone.

- The three solution steps (liquid-oriented mechanics, solid-oriented mechanics, and heat transfer), are performed sequentially for each time step on the same global mesh. For each solution step, conservation equations are solved in a general arbitrary Lagrangian-Eulerian (ALE) framework, with a level-set formulation to track the free surface evolution at the meniscus.

700 The correct implementation of this algorithm was firstly checked through a simple verification test case.

701 It was then applied to a 3D pilot CC process. Simulation gave promising results, in which fluid flow in the bulk liquid or mushy zone and stress formed in the solid regions were concomitantly computed.

703 The main perspectives of this work are twofold. Firstly, coupling this new algorithm with

704 macrosegregation modeling would allow demonstrating the influence of solid deformation on central macrosegregation in CC processes. ${ }^{[1]}$ Secondly, the coupling with equiaxed grain motion would provide access to enhanced predictions of macrosegregation due to thermo-solutal convection, shrinkage flow and transport of equiaxed grains. ${ }^{[27]}$ 


\section{Acknowledgments}

710

711

712

713

714

715

716

717

718

719

720

721

722

723

724

725

726

727

728

729

730

731

732

733

734

735

736

737

738

739

740

741

742

743

744

745

746

747

748

749

750

751

752

753

754

755

756

757

This work was funded by the European Space Agency - ESTEC (Netherlands) under the projects CCEMLCC (Grant AO-2004-017) and industrial partners ARCELORMITTAL Maizières Research, APERAM ALLOYS IMPHY and INDUSTEEL.

The authors would also like to thank Prof. Brian G. Thomas (Colorado School of Mines, and University of Illinois), for fruitful exchanges during the revision of this manuscript.

\section{References}

[1] B.G. Thomas, L.J. Mika and F.M. Najjar: Metall. Trans. B, 1990, vol. 21B, pp. 387-400.

[2] F.M. Najjar, B.G. Thomas and D.E. Hershey: Metall. Mater. Trans. B, 1995, vol. 26B, pp. 74965.

[3] R. Vertnik and B. Šarler: Eng. Anal. with Boundary Elements, 2014, vol.45, pp. 45-61.

[4] C. Pfeiler, M. Wu and A. Ludwig: Mater. Sci. Eng. A, 2005, vol. 413, pp. 115-20.

[5] P.E. Ramirez-Lopez, P.D. Lee and K.C. Mills: ISIJ Int., 2010, vol. 50, pp. 425-34.

[6] M.R. Aboutalebi, M. Hasan and R.I.L. Guthrie: Metall. Mater. Trans. B, 1995, vol. 26, pp. 731-44.

[7] A. Noeppel, A. Ciobanas, X.D. Wang, K. Zaidat, N. Mangelinck, O. Budenkova, A. Weiss, G. Zimmermann and Y. Fautrelle: Metall. Mater. Trans. B, 2010, vol. 41, pp. 193-208.

[8] F. Pascon and A.-M. Habraken: Comput. Meth. Appl. Mech. Eng., 2007, vol.196, pp. 2285-99.

[9] L.C. Hibbeler, B.G. Thomas, R.C. Schimmel and G. Abbel: Metall. Mater. Trans. B, 2012, vol. 43, pp. 1156-72.

[10] M. Bellet and A. Heinrich: ISIJ Int., 2004, vol. 44, pp. 1686-95.

[11] T. Koshikawa, M. Bellet, C.-A. Gandin, H. Yamamura and M. Bobadilla: Acta Mater., 2017,vol. 124, pp. 513-27.

[12] M.L.S. Zappulla, S.-M. Cho, S. Koric, H.-J. Lee, S.-H. Kim, B.G. Thomas: Journal of Materials Processing Tech., 2020, vol. 278, 116469.

[13] S. Koric, L.C. Hibbeler, R. Liu, B.G. Thomas: Numerical Heat Transfer, Part B, 2010, vol. 58: pp. 371-92.

[14] M. Heil, A.L. Hazel and J. Boyle: Comput. Mech., 2008, vol. 43, pp. 91-101.

[15] S. Zhang, G. Guillemot, C.-A. Gandin and M. Bellet: Comput. Meth. Appl. Mech. Eng.,2019, vol. 356, pp. 294-324.

[16] V.D. Fachinotti, S. Le Corre, N. Triolet, M. Bobadilla and M. Bellet: Int. J. Num. Meth. Eng., 2006, vol. 67, pp. 1341-84.

[17] A. Ludwig, A. Vakhrushev, M. Wu, T. Holzmann and A. Kharicha: Trans. Indian Instit. Metals, 2015, vol. 68, pp. 1087-94.

[18] C.M.G Rodrigues, A. Ludwig, M. Wu, A. Kharicha and A. Vakhrushev: Metall. Mater. Trans. B, 2019, vol. 50B, pp. 1334-50.

[19] M. Bellet and B.G. Thomas: Materials Processing Handbook, CRC Press, Taylor and Francis, 2007, Chapter 27, pp. 27-1 - 27-26.

[20] A. Saad, C.-A. Gandin and M. Bellet: Comput. Mater. Sci., 2015, vol. 99, pp. 221-31.

[21] J. Ni and C. Beckermann: Metall. Trans. B, 1991, vol. 22, pp. 349-61.

[22] P.C. Carman: Chem. Eng. Res. Design: Trans. Instit. Chem. Eng. Part A, 1937, vol. 37, pp. 206979.

[23] E. Hachem, B. Rivaux, T. Kloczko, H. Digonnet and T. Coupez: J. Comp. Phys., 2010, vol. 229, pp. 8643-65.

[24] M. Shakoor, B. Scholtes, P.O. Bouchard and M. Bernacki: Appl. Math. Model., 2015, vol. 39, pp. 7291-302. 
[25] T. Coupez: J. Comp. Phys., 2011, vol. 230, pp. 2391-405.

[26] M. Henri: Modélisation 3D par éléments finis du refroidissement primaire lors de la coulée continue d'aciers (3D Finite Element Modeling of primary cooling during steel continuous casting), Ph.D. Thesis (in French), Ecole Nationale Supérieure des Mines de Paris, 2009.

[27] T.T.M. Nguyen, C.-A. Gandin, H. Combeau, M. Založnik and M. Bellet: Metall. Mater. Trans. A, 2018, vol. 49A, pp. 1725-48.

[28] B.G. Thomas, R. O'Malley, D. Stone: MCWASP VIII Conf. Proc., 1998, TMS, p.1185.

[29] C. Pfeiler, B.G. Thomas, M. Wu, A. Ludwig, A. Kharicha: Steel Research Int., 2006, vol. 77, No.7

[30] J.M. Risso, A.E. Huespe, A. Cardona: International Journal for Numerical Methods in Engineering, 2006, vol. 65, pp. 1355-77.

[31] M.L.S. Zappulla, L.C. Hibbeler, B.G. Thomas: Metallurgical and Materials Transactions A, 2017, vol. 48, pp. 3777-93. 